Nilpotent commuting varieties of reductive Lie algebras

Premet, Alexander

2003

MIMS EPrint: 2006.288

Manchester Institute for Mathematical Sciences

School of Mathematics

The University of Manchester

\footnotetext{
Reports available from: http://eprints.maths.manchester.ac.uk/

And by contacting: The MIMS Secretary

School of Mathematics

The University of Manchester

Manchester, M13 9PL, UK
} 


\title{
NILPOTENT COMMUTING VARIETIES OF REDUCTIVE LIE ALGEBRAS
}

\author{
ALEXANDER PREMET
}

\begin{abstract}
Let $G$ be a connected reductive algebraic group over an algebraically closed field $k$ of characteristic $p \geq 0$, and $\mathfrak{g}=\operatorname{Lie} G$. In positive characteristic, suppose in addition that $p$ is good for $G$ and the derived subgroup of $G$ is simply connected. Let $\mathcal{N}=\mathcal{N}(\mathfrak{g})$ denote the nilpotent variety of $\mathfrak{g}$, and $\mathfrak{C}^{\text {nil }}(\mathfrak{g}):=\{(x, y) \in$ $\mathcal{N} \times \mathcal{N} \mid[x, y]=0\}$, the nilpotent commuting variety of $\mathfrak{g}$. Our main goal in this paper is to show that the variety $\mathfrak{C}^{\text {nil }}(\mathfrak{g})$ is equidimensional. In characteristic 0 , this confirms a conjecture of Vladimir Baranovsky; see [2]. When applied to GL $(n)$, our result in conjunction with an observation in [2] shows that the punctual (local) Hilbert scheme $\mathcal{H}_{n} \subset \operatorname{Hilb}^{n}\left(\mathbb{P}^{2}\right)$ is irreducible over any algebraically closed field.
\end{abstract}

\section{Introduction}

Let $k$ be an algebraically closed field of characteristic $p \geq 0$. The purpose of this note is to confirm Baranovsky's conjecture [2, p. 4] which states that all irreducible components of the nilpotent commuting variety $\mathfrak{C}^{\text {nil }}(\mathfrak{g})$ of a complex semisimple Lie algebra $\mathfrak{g}$ have the same dimension, equal to $\operatorname{dim} \mathfrak{g}$, and are parametrised by the distinguished nilpotent orbits in $\mathfrak{g}$. The main result in [2] confirmed the conjecture for $\mathfrak{g}=\mathfrak{s l}(n)$ (an earlier proof of the irreducibility of $\mathfrak{C}^{\text {nil }}(\mathfrak{s l}(n))$ in [12] was incomplete). Notably, it is observed in [2] that in any characteristic the number of irreducible components of $\mathfrak{C}^{\text {nil }}(\mathfrak{s l}(n))$ equals the number of irreducible components of the punctual Hilbert scheme $\mathcal{H}_{n} \subset \operatorname{Hilb}^{n}\left(\mathbb{P}^{2}\right)$; see [2, Remark 1].

The punctual Hilbert scheme $\mathcal{H}_{n}$ parametrises the ideals of colength $n$ in the ring of formal power series $k[[x, y]]$. It has been known for more than 25 years that the scheme $\mathcal{H}_{n}$ is irreducible in characteristic 0, thanks to the work of Briançon [6]. This was extended to the case where $p>n$ by Iarrobino [14]; see also [11]. These results enabled Baranovsky to deduce that $\mathfrak{C}^{\text {nil }}(\mathfrak{s l}(n))$ is irreducible for $p=0$ and $p>n$. It should be mentioned here that very recently a more direct proof of the irreducibility of $\mathfrak{C}^{\text {nil }}(\mathfrak{s l}(n))$ was found by Basili in [1]. It works for $p \geq n / 2$ and $p=0$ implying the irredicibility of $\mathcal{H}_{n}$ for $p$ in that range.

In this note we give a direct proof of Baranovsky's conjecture entirely in the framework of Lie Theory. In view of [2, Remark 1], this will enable us to conclude that $\mathcal{H}_{n}$ is irreducible over any algebraically closed field. For $p<n / 2$, this appears to be a new result in Algebraic Geometry. Our approach also provides a much shorter and more elementary proof of the irreducibility of $\mathcal{H}_{n}$ over $\mathbb{C}$. In principle, it can be used for investigating the connected components of $\mathcal{H}_{n}$ over other locally compact fields; see [14] and [2, Remark 2].

Let $G$ be a connected reductive algebraic group over $k$ and $\mathfrak{g}=$ Lie $G$. We assume throughout the paper that the derived subgroup $(G, G)$ is simply connected and $p$

Mathematics Subject Classification (1985 revision). Primary 20 G05. 
is good for the root system of $G$. When $p>0$, the Lie algebra $\mathfrak{g}$ carries a natural (Ad $G$ )-equivariant $[p]$-mapping $x \mapsto x^{[p]}$. In this case it is well-known that $e \in \mathfrak{g}$ is nilpotent if and only if $e^{[p]^{N}}=0$ for $N$ large enough. When $p=0$, an element $e \in \mathfrak{g}$ is nilpotent if and only if $e \in[\mathfrak{g}, \mathfrak{g}]$ and the endomorphism ad $e$ is nilpotent. The variety of all nilpotent elements in $\mathfrak{g}$ is denoted by $\mathcal{N}$. It is well-known that $\mathcal{N}$ is an irreducible Zariski closed subset in $\mathfrak{g}$ of dimension $\operatorname{dim} G-\operatorname{rk} G$ and $G$ acts on $\mathcal{N}$ with finitely many orbits; see [30]. Moreover, the Bala-Carter theory holds in good characteristic and the $(\operatorname{Ad} G)$-orbits in $\mathcal{N}$ are described in the same way as over $\mathbb{C}$. In other words, any nilpotent element in $\mathfrak{g}$ is $(\operatorname{Ad} G)$-conjugate to a Richardson element in a distinguished parabolic subalgebra of Lie $L$ where $L$ is a Levi subgroup of $G$; see $[7,23,24,29]$. Let

$$
\mathfrak{C}^{\text {nil }}(\mathfrak{g}):=\{(x, y) \in \mathcal{N} \times \mathcal{N} \mid[x, y]=0\} \subset \mathfrak{g} \times \mathfrak{g},
$$

the nilpotent commuting variety of $\mathfrak{g}$. Obviously, the Zariski closed set $\mathfrak{C}^{\text {nil }}(\mathfrak{g})$ is preserved by the diagonal action of $G$ on $\mathfrak{g} \times \mathfrak{g}$.

Given a closed subgroup $H$ in $G$ we denote by $Z_{H}(e)$ the centraliser of $e$ in $H$. As usual we denote by $R_{u}(H)$ the unipotent radical of $H$. An element $e \in \mathcal{N}$ is called distinguished if the connected component of $Z_{(G, G)}(e)$ is unipotent, that is if Lie $Z_{(G, G)}(e) \subset \mathcal{N}$. According to the main result of the Bala-Carter theory, any distinguished nilpotent element in $\mathfrak{g}$ is Richardson in a distinguished parabolic subalgebra of $\mathfrak{g}$. Note that for any distinguished $e \in \mathcal{N}$ we have $\left(e\right.$, Lie $\left.Z_{(G, G)}(e)\right) \subset \mathfrak{C}^{\text {nil }}(\mathfrak{g})$. We denote by $\mathfrak{C}(e)$ the Zariski closure of $G \cdot\left(e\right.$, Lie $\left.Z_{(G, G)}(e)\right)$ in $\mathfrak{C}^{\text {nil }}(\mathfrak{g})$. Our main result in this paper is the following:

Theorem. Let $e_{1}, \ldots, e_{r}$ be representatives of the distinguished nilpotent orbits in $\mathfrak{g}$. The closed sets $\mathfrak{C}\left(e_{1}\right), \ldots, \mathfrak{C}\left(e_{r}\right)$ are pairwise distinct and all have the same dimension equal to $\operatorname{dim}(G, G)$. Moreover, $\mathfrak{C}^{\text {nil }}(\mathfrak{g})=\mathfrak{C}\left(e_{1}\right) \cup \ldots \cup \mathfrak{C}\left(e_{r}\right)$.

A well-known result of Richardson [31] asserts that for $p=0$ the whole commuting variety $\mathfrak{C}(\mathfrak{g}):=\{(x, y) \in \mathfrak{g} \times \mathfrak{g} \mid[x, y]=0\}$ of $\mathfrak{g}$ coincides with the Zariski closure of $G \cdot($ Lie $T \times$ Lie $T$ ) in $\mathfrak{g} \times \mathfrak{g}$ where $T$ is a maximal torus in $G$. As a consequence, this variety is always irreducible. The case where $p$ is good was recently settled by P. Levy in his PhD thesis; see also [19]. An important long-standing conjecture asserts that the variety $\mathfrak{C}(\mathfrak{g})$ is always normal and the ideal defining $\mathfrak{C}(\mathfrak{g})$ is given by the "obvious" quadratic equations. It is not hard to see that $\mathfrak{C}^{\text {nil }}(\mathfrak{g})$ coincides with the special fibre of the quotient map $\mathfrak{C}(\mathfrak{g}) \rightarrow \mathfrak{C}(\mathfrak{g}) / / G$. For $p=0$, a Chevalley Restriction Theorem holds for $\mathfrak{C}(\mathfrak{g})[17]$; it says that the affine variety $\mathfrak{C}(\mathfrak{g}) / / G$ is isomorphic to $(\operatorname{Lie} T \times \operatorname{Lie} T) / / W$ where the action of the Weyl group $W=N_{G}(T) / T$ on Lie $T \times$ Lie $T$ is induced by the diagonal action of $G$ on $\mathfrak{g} \times \mathfrak{g}$. It would be useful to have an analogue of this in positive characteristic.

To emphasize the elementary nature of our proof we first consider the case where $p=0$ (this is done in Section 2). In the modular case our argument goes along the same lines but is technically much more involved (see Section 3). This is mainly due to inseparability problems and a rather unusual behaviour of the orbit map: in small characteristic there exist nontrivial commuting $\mathfrak{s l}_{2}$-triples $\left(e_{1}, h_{1}, f_{1}\right)$ and $\left(e_{2}, h_{2}, f_{2}\right)$ in $\mathfrak{g}$ such that $e_{1}$ and $e_{1}+e_{2}$ are conjugate under the adjoint action of $G$. The latter is, in our opinion, the main reason why the irredicibility of $\mathcal{H}_{n}$ is harder to establish for 
$p$ small. To tackle this problem we go case-by-case and look closely at the centralisers of nilpotent elements. For exceptional types, we have to use the computations in [27].

As a motivation for further study, we mention that the nilpotent commuting variety and its higher analogues play a very important rôle in the cohomology theory of the Frobenius kernels of $G$. It is proved in [37] that $\mathfrak{C}^{\text {nil }}(\mathfrak{g})$ is homeomorphic to the spectrum of maximal ideals of the Yoneda algebra $\bigoplus_{i>0} H^{2 i}\left(G_{2}, k\right)$ of the second Frobenius kernel $G_{2}$ of $G$ provided that $p$ is sufficiently large. The variety $\mathfrak{C}^{\text {nil }}(\mathfrak{g})$ is also important for the study of support varieties of modules over reduced enveloping algebras of $\mathfrak{g}$; see [28].

In Section 4, we prove that the punctual Hilbert scheme $\mathcal{H}_{n}$ is irreducible over any algebraically closed field; see Corollary 4.1. We also show, in (4.2), that the nilpotent variety of any finite dimensional restricted Lie algebra is equidimensional. This result is then used in (4.3) to estimate the dimension of $\mathfrak{C}^{\text {nil }}(\mathfrak{g})$ in the case where $p$ is a bad prime for $G$.

Acknowledgement. I would like to thank V. Ginzburg, J.C. Jantzen, D. Panyushev, D. Timashev and È.B. Vinberg for their interest, comments and informations, and the referee for some very useful suggestions. I am also grateful to P. Levy from whom I learned that $\mathrm{GL}(2)$ acts on the commuting variety $\mathfrak{C}(\mathfrak{g})$.

\section{The classical case}

2.1. Unless otherwise indicated we assume in this section that $p=0$. In this case, $\mathfrak{g}=[\mathfrak{g}, \mathfrak{g}] \oplus \mathfrak{z}$ where $\mathfrak{z}$ is the Lie algebra of the connected centre of $G$. Thus no generality will be lost by assuming that $G$ is semisimple. Then $G$ and $\mathfrak{g}$ are both defined over $\mathbb{Q}$ and hence so is $\mathfrak{C}^{\text {nil }}(\mathfrak{g})$. Therefore, all irreducible components of $\mathfrak{C}^{\text {nil }}(\mathfrak{g})$ are defined over the field of algebraic numbers. Thus it can be assumed in what follows that $k=\mathbb{C}$.

Let $e$ be a nilpotent element in $\mathfrak{g}$. Let $\mathfrak{z}(e)$ denote the centraliser of $e$ in $\mathfrak{g}$. By the Jacobson-Morozov theorem, $e$ can be embedded into an $\mathfrak{s l}_{2}$-triple $(e, h, f)$ in $\mathfrak{g}$. By the $\mathfrak{s l}_{2}$-theory, all eigenvalues of the semisimple endomorphism ad $h$ are integers. For $i \in \mathbb{Z}$ we let $\mathfrak{g}(i ; h)=\{x \in \mathfrak{g} \mid[h, x]=i x\}$. Then $\mathfrak{g}=\bigoplus_{i \in \mathbb{Z}} \mathfrak{g}(i ; h)$ and $[\mathfrak{g}(i ; h), \mathfrak{g}(j ; h)] \subseteq \mathfrak{g}(i+j ; h)$ for all $i, j \in \mathbb{Z}$.

Since all nonzero elements in $\mathfrak{z}(e ; i):=\mathfrak{g}(i ; h) \cap \mathfrak{z}(e)$ are heighest weight vectors for $\mathfrak{s}:=\mathbb{C} e \oplus \mathbb{C} h \oplus \mathbb{C} f$ we have the inclusion $\mathfrak{z}(e) \subset \bigoplus_{i \geq 0} \mathfrak{g}(i ; h)$ (again, by the $\mathfrak{s l}_{2}$-theory). The Lie algebra $\mathfrak{z}(e ; 0)$ is nothing but the centraliser of $\mathfrak{s}$ in $\mathfrak{g}$ hence reductive. Therefore, the ideal $\bigoplus_{i>0} \mathfrak{z}(e ; i)$ of $\mathfrak{z}(e)$ coincides with the nilradical of $\mathfrak{z}(e)$. As a consequence, the variety

$$
\mathcal{N} \cap \mathfrak{z}(e)=\mathcal{N}(\mathfrak{z}(e ; 0))+\operatorname{nil} \mathfrak{z}(e) \cong \mathcal{N}(\mathfrak{z}(e ; 0)) \times \operatorname{nil} \mathfrak{z}(e)
$$

is irreducible. We denote by $\mathfrak{C}(e)$ the Zariski closure of $G \cdot(e, \mathcal{N} \cap \mathfrak{z}(e))$ in $\mathfrak{g} \times \mathfrak{g}$. It is immediate from the definition that $\mathfrak{C}(e) \subseteq \mathfrak{C}^{\text {nil }}(\mathfrak{g})$ and $\mathfrak{C}(e)=\mathfrak{C}((\operatorname{Ad} g) e)$ for any $g \in G$. The preceding remark shows that each $\mathfrak{C}(e)$ is irreducible. The morphism

$$
\xi: G \times(N \cap \mathfrak{z}(e)) \longrightarrow \mathfrak{C}(e), \quad \xi(g, x)=((\operatorname{Ad} g) e,(\operatorname{Ad} g) x),
$$

is dominant and the fibre $\xi^{-1}(\xi(g, x))$ is just the set of all pairs $\left(a,\left(\operatorname{Ad} a^{-1} g\right) x\right)$ with $g^{-1} a \in Z_{G}(e)$. Hence $\xi^{-1}(\xi(g, x)) \cong Z_{G}(e)$ as varieties. The theorem on the 
dimension of the fibres of a morphism now gives

$$
\operatorname{dim} \mathfrak{C}(e)=\operatorname{dim} G+\operatorname{dim} \mathcal{N} \cap \mathfrak{z}(e)-\operatorname{dim} Z_{G}(e)=n-\operatorname{rk} \mathfrak{z}(e ; 0) .
$$

As a consequence, $\operatorname{dim} \mathfrak{C}(e) \leq n$ and $\operatorname{dim} \mathfrak{C}(e)=n$ if and only if $e$ is distinguished.

Definition. We call a nilpotent element $e \in \mathfrak{g}$ almost distinguished if the centraliser of $e$ in $\mathfrak{g}$ is a solvable Lie algebra.

Note that $e$ is almost distinguished if and only if the connected component of the centraliser $Z_{G}(e)$ is solvable. Any distinguished nilpotent element in $\mathfrak{g}$ is therefore almost distinguished. The converse, however, holds only for simple algebraic groups of small rank. Looking through the tables in [7, Chap. 13] one finds out that the exceptional groups for which the converse also holds are the groups of types $\mathrm{G}_{2}$ and $\mathrm{F}_{4}$. At the other extreme, the Lie algebra $\mathfrak{s l}(n)$ has only one distinguished nilpotent orbit while there is a bijection between the almost distinguished nilpotent orbits in $\mathfrak{s l}(n)$ and the partitions of $n$ with pairwise distinct parts.

Since $\mathfrak{z}(e ; 0)$ is reductive, the Lie algebra $\mathfrak{z}(e)$ is solvable if and only if $\mathfrak{z}(e ; 0)$ coincides with the Lie algebra of a maximal torus in $Z_{G}(e)$. From this it is immediate that $e \in \mathcal{N}$ is almost distinguished if and only if $\mathcal{N} \cap \mathfrak{z}(e)=\bigoplus_{i>0} \mathfrak{z}(e ; i)$.

Proposition 2.1. Each irreducible component of $\mathfrak{C}^{\text {nil }}(\mathfrak{g})$ is of the form $\mathfrak{C}(e)$ for some almost distinguished $e \in \mathfrak{g}$.

Proof. (1) The group GL(2) acts on $\mathfrak{g} \times \mathfrak{g}$ via

$$
\left(\begin{array}{ll}
\alpha & \beta \\
\gamma & \delta
\end{array}\right) \cdot(x, y)=(\alpha x+\beta y, \gamma x+\delta y)
$$

As any linear combination of two commuting elements in $\mathcal{N}$ is again in $\mathcal{N}$, the variety $\mathfrak{C}^{\text {nil }}(\mathfrak{g})$ is $\mathrm{GL}(2)$-invariant. As $\mathrm{GL}(2)$ is a connected group, it fixes each irreducible component of $\mathfrak{C}^{\text {nil }}(\mathfrak{g})$. In particular, each irreducible component of $\mathfrak{C}^{\text {nil }}(\mathfrak{g})$ is invariant under the involution $\sigma:(x, y) \mapsto(y, x)$ on $\mathfrak{g} \times \mathfrak{g}$.

(2) Let $e_{1}, \ldots, e_{s}$ be representatives of the nilpotent orbits in $\mathfrak{g}$. Since each $\mathfrak{C}\left(e_{i}\right)$ is $G$-stable we have that $\mathfrak{C}^{\text {nil }}(\mathfrak{g})=\mathfrak{C}\left(e_{1}\right) \cup \ldots \cup \mathfrak{C}\left(e_{s}\right)$. This implies that each irreducible component of $\mathfrak{C}^{\text {nil }}(\mathfrak{g})$ has the form $\mathfrak{C}\left(e_{i}\right)$ for some $i \leq s$. Let $e \in \mathcal{N}$ be such that $\mathfrak{C}(e)$ is a component of $\mathfrak{C}^{\text {nil }}(\mathfrak{g})$. By part 1 , the set $\mathfrak{C}(e)$ is then $\sigma$-stable.

Let $\mathcal{O}$ denote the $G$-orbit of $e$. The map $\pi:(x, y) \mapsto x$ takes $G \cdot(e, \mathcal{N} \cap \mathfrak{z}(e))$ onto $\mathcal{O}$, hence $\mathfrak{C}(e)=\overline{G \cdot(e, \mathcal{N} \cap \mathfrak{z}(e))}$ onto the Zariski closure $\overline{\mathcal{O}} \subset \mathfrak{g}$. This shows that

$$
\mathcal{N} \cap \mathfrak{z}(e)=(\pi \circ \sigma)(e, \mathcal{N} \cap \mathfrak{z}(e)) \subset \overline{\mathcal{O}} .
$$

(3) We need to show that the element $e$ is almost distinguished. So suppose the contrary. Then $\mathfrak{z}(e ; 0)$ contains a nonzero nilpotent element, say $e_{0}$. Note that $e+e_{0} \in$ $\mathcal{N} \cap \mathfrak{z}(e)$ so that $e+e_{0} \in \overline{\mathcal{O}}$, by part 2 . Since $\mathfrak{z}(e ; 0)$ is reductive, $e_{0}$ can be included into an $\mathfrak{s l}_{2}$-triple $\left(e_{0}, h_{0}, f_{0}\right)$ in $\mathfrak{z}(e ; 0)$, again by the Jacobson-Morozov theorem. Let $\mathfrak{s}_{0}=\mathbb{C} e_{0} \oplus \mathbb{C} h_{0} \oplus \mathbb{C} f_{0}$. Since $\mathfrak{s}_{0} \subseteq \mathfrak{z}(e ; 0)$, the Lie subalgebras $\mathfrak{s}$ and $\mathfrak{s}_{0}$ commute with each other. This enables us to deduce that $\left(e+e_{0}, h+h_{0}, f+f_{0}\right)$ is an $\mathfrak{s l}_{2}$-triple in g. Applying the automorphisms $\exp \left(\lambda \operatorname{ad}\left(h+h_{0}\right)\right) \circ \exp \left(\lambda^{-1}\right.$ ad $\left.h\right)$ from the adjoint group of $\mathfrak{g}$ to $e+e_{0}$ we deduce that $e+\mathbb{C}^{*} e_{0} \subset \mathcal{O}\left(e+e_{0}\right)$, the $G$-orbit of $e+e_{0}$. Then $e \in \overline{\mathcal{O}\left(e+e_{0}\right)}$. As a result, $\overline{\mathcal{O}\left(e+e_{0}\right)}=\overline{\mathcal{O}}$ showing that $e$ and $e+e_{0}$ are $G$-conjugate. But then the semisimple elements $h$ and $h+h_{0}$ are $G$-conjugate too; see [7, Prop. 5.6.4] for example. 
(4) Since $\mathfrak{g}(0 ; h)$ is reductive we have that $\mathfrak{g}(0 ; h)=[\mathfrak{g}(0 ; h), \mathfrak{g}(0 ; h)] \oplus \mathfrak{z}^{\prime}$ where $\mathfrak{z}^{\prime}$ is the centre of $\mathfrak{g}(0 ; h)$. The subspaces $\mathfrak{z}^{\prime}$ and $[\mathfrak{g}(0 ; h), \mathfrak{g}(0 ; h)]$ are orthogonal to each other with respect to the Killing form $\kappa$ of $\mathfrak{g}$. Since $\mathfrak{s}_{0} \subseteq[\mathfrak{g}(0 ; h), \mathfrak{g}(0 ; h)]$ and $h \in \mathfrak{z}^{\prime}$ it must be that $\kappa\left(h, h_{0}\right)=0$. On the other hand, $\kappa(h, h)>0$ and $\kappa\left(h_{0}, h_{0}\right)>0$ as all eigenvalues of ad $h$ and ad $h_{0}$ are in $\mathbb{Z}$. But then

$$
\kappa\left(h+h_{0}, h+h_{0}\right)=\kappa(h, h)+\kappa\left(h_{0}, h_{0}\right)>\kappa(h, h) .
$$

Since $h$ and $h+h_{0}$ are $G$-conjugate (see part 3) this is impossible. By contradiction, the proposition follows.

2.2. In a sense, our quest requires that we attach to an arbitrary $\mathfrak{s l}_{2}$-triple $(e, h, f)$ in $\mathfrak{g}$ a nice $\mathfrak{s l}_{2}$-triple $(\tilde{e}, \tilde{h}, \tilde{f})$ with $\tilde{e}$ distinguished. This will be achieved with the help of the Bala-Carter theory; see [7, Chap. 5] and [29]. In this subsection, we work with an arbitrary reductive group $G$ over $k$ assuming only that $p=\operatorname{char} k$ is good for $G$.

Fix a maximal torus $T$ in $G$ and let $\Phi$ denote the root system of $G$ relative to $T$. Let $\Pi=\left\{\alpha_{1}, \ldots, \alpha_{l}\right\}$ be a basis of simple roots in $\Phi, \Phi^{+}$be the positive system in $\Phi$ associated with $\Pi$, and $X_{*}(T)$ be the lattice of cocharacters of $T$ (the latter contains all coroots $\alpha^{\vee}$ ). For a subset $I \subseteq\{1, \ldots, l\}$ let $L_{I}$ (resp., $P_{I}$ ) stand for the standard Levi (respectively, parabolic) subgroup of $G$ corresponding to $I$. Let $\Phi_{I}$ be the root system of $L_{I}$ relative to $T$. The set $\Pi_{I}:=\left\{\alpha_{i} \mid i \in I\right\}$ is then a basis of simple roots in $\Phi_{I}$.

Given two subsets $I \supseteq J$ in $\{1, \ldots, l\}$ we denote by $P_{I, J}$ the standard parabolic subgroup of $L_{I}$ associated with $J$. Let $\mathfrak{l}_{I}=$ Lie $L_{I}, \mathfrak{p}_{I}=$ Lie $P_{I}, \mathfrak{p}_{I, J}=$ Lie $P_{I, J}$, and $\mathfrak{u}_{I, J}=$ Lie $R_{u}\left(P_{I, J}\right)$. Note that $P_{I, J}=L_{J} \cdot R_{u}\left(P_{I, J}\right)$ is a Levi decomposition in $P_{I, J}$. According to $[7$, Prop. 5.8.2], for each pair $(I, J)$ with $\{1, \ldots, l\} \supseteq I \supseteq J$ the inequality

$$
\operatorname{dim}\left(L_{I}, L_{I}\right) \cap L_{J} \geq \operatorname{dim} R_{u}\left(P_{I, J}\right) /\left(R_{u}\left(P_{I, J}\right), R_{u}\left(P_{I, J}\right)\right)
$$

holds. A subgroup $P_{I, J}$ is said to be distinguished in $L_{I}$ if

$$
\operatorname{dim}\left(L_{I}, L_{I}\right) \cap L_{J}=\operatorname{dim} R_{u}\left(P_{I, J}\right) /\left(R_{u}\left(P_{I, J}\right), R_{u}\left(P_{I, J}\right)\right) .
$$

Let $\mathcal{P}(\Pi)$ be the set of all pairs $(I, J)$ with $\{1, \ldots, l\} \supseteq I \supseteq J$ such that $P_{I, J}$ is distinguished in $L_{I}$. For $(I, J) \in \mathcal{P}(\Pi)$ we denote by $\mathcal{O}(I, J)$ the nilpotent orbit in $\mathfrak{g}$ containing a Richardson element in $\mathfrak{u}_{I, J}$. The main result of the Bala-Carter theory (as extended to the present case in $[23,24,29]$ ) asserts that $\mathcal{N}=\bigcup_{(I, J) \in \mathcal{P}(\Pi)} \mathcal{O}(I, J)$. Thus we may assume in what follows that $e$ is a Richardson element in $\mathfrak{u}_{I, J}$.

Given $\mu \in X_{*}(T)$ and a $\mu\left(k^{*}\right)$-invariant subalgebra $\mathfrak{m}$ in $\mathfrak{g}$ we denote by $\mathfrak{m}(i ; \mu)$ the subspace of all $x \in \mathfrak{m}$ such that $(\operatorname{Ad} \mu(t)) x=t^{i} x$ for all $t \in k^{*}$ (here $i \in \mathbb{Z}$ ). As explained in [29, Sect. 2] (for example) there exists a cocharacter $\lambda_{I, J} \in \sum_{i \in I} \mathbb{Z} \alpha_{i}^{\vee}$ such that

$$
\alpha_{i}\left(\lambda_{I, J}(t)\right)= \begin{cases}1 & \text { if } i \in J, \\ t^{2} & \text { if } i \in I \backslash J\end{cases}
$$

for all $t \in k^{*}$. When $I=\{1, \ldots, l\}$ (that is when $P_{J}$ is distinguished in $G$ ) we denote the cocharacter $\lambda_{I, J}$ by $\lambda_{J}$. Since the orbit $\left(\operatorname{Ad} P_{I, J}\right)$ e meets $\mathfrak{l}_{I}\left(2 ; \lambda_{I, J}\right)$, by $[7$, Prop. 5.8.5], we may (and will) assume that $e \in \mathfrak{l}_{I}\left(2 ; \lambda_{I, J}\right)$.

Our next result is implicit in [20]. We give a direct proof for the benefit of the reader. 
Lemma 2.2 (cf. [20, Prop. 1.12]). For each $(I, J) \in \mathcal{P}(\Pi)$ the parabolic subgroup $P_{J}$ is distinguished in $G$.

Proof. Following $[7,(5.8)]$ define a function $\eta_{J}: \Phi \rightarrow 2 \mathbb{Z}$ by

$$
\eta_{J}\left(\alpha_{i}\right)= \begin{cases}0 & \text { if } i \in J \\ 2 & \text { if } i \in\{1, \ldots, l\} \backslash J\end{cases}
$$

and extending to arbitrary roots by linearity. Applying [7, Prop. 5.8.1] to $P_{J} \subset G$ and $P_{I, J} \subset L_{I}$ and using the fact that $(I, J) \in \mathcal{P}(\Pi)$ we deduce that

$$
\begin{aligned}
\operatorname{dim} R_{u}\left(P_{J}\right) /\left(\left(R_{u}\left(P_{J}\right), R_{u}\left(P_{J}\right)\right)\right. & =\operatorname{Card}\left\{\alpha \in \Phi \mid \eta_{J}(\alpha)=2\right\} \\
& \geq\left|\Pi \backslash \Pi_{I}\right|+\operatorname{Card}\left\{\alpha \in \Phi_{I} \mid \eta_{J}(\alpha)=2\right\} \\
& =l-|I|+\operatorname{dim} R_{u}\left(P_{I, J}\right) /\left(\left(R_{u}\left(P_{I, J}\right), R_{u}\left(P_{I, J}\right)\right)\right. \\
& =l-|I|+\operatorname{dim}\left(L_{I}, L_{I}\right) \cap L_{J} .
\end{aligned}
$$

On the other hand, $\operatorname{dim}(G, G) \cap L_{J}=l+\left|\Phi_{J}\right|=l-|I|+\operatorname{dim}\left(L_{I}, L_{I}\right) \cap L_{J}$. Hence

$$
\operatorname{dim} R_{u}\left(P_{J}\right) /\left(\left(R_{u}\left(P_{J}\right), R_{u}\left(P_{J}\right)\right) \geq \operatorname{dim}(G, G) \cap L_{J} .\right.
$$

Applying (1) with $I=\{1, \ldots, l\}$ yields that $P_{J}$ is distinguished in $G$ as desired.

2.3. From now we assume in this section that $k=\mathbb{C}$ and $\mathfrak{g}$ is semisimple. Let $(I, J) \in \mathcal{P}(\Pi)$ and let $e \in \mathfrak{l}_{I}\left(2 ; \lambda_{I, J}\right)$ be such that the orbit $\left(\operatorname{Ad} P_{I, J}\right) e$ is open in $\mathfrak{u}_{I, J}$. It is immediate from $[7$, Cor. 5.2.4] that the map

$$
\operatorname{ad} e: \mathfrak{l}_{I}\left(-2 ; \lambda_{I, J}\right) \longrightarrow\left[\mathfrak{l}_{I}, \mathfrak{l}_{I}\right]\left(0 ; \lambda_{I, J}\right)
$$

is bijective. This implies that there are $h \in \operatorname{Lie} \lambda_{I, J}\left(\mathbb{C}^{*}\right)$ and $f \in \mathfrak{l}_{I}\left(-2 ; \lambda_{I, J}\right)$ such that $(e, h, f)$ is an $\mathfrak{s l}_{2}$-triple in $\mathfrak{g}$. Moreover, $\alpha_{i}(h)=0$ for $i \in J$ and $\alpha_{i}(h)=2$ for $i \in I \backslash J$.

By Lemma 2.2, $P_{J}$ is a distinguished parabolic subgroup of $G$. Then $\lambda_{J} \in X_{*}(T)$ is well-defined and $\mathfrak{p}_{J}=\bigoplus_{i>0} \mathfrak{g}\left(2 i ; \lambda_{J}\right)$. By [7, Prop. 5.8.5], the subspace $\mathfrak{g}\left(2 ; \lambda_{J}\right)$ contains a Richardson element of $\mathfrak{p}_{J}$. We pick such an element and call it $\tilde{e}$. There is $\tilde{h} \in$ Lie $\lambda_{J}\left(\mathbb{C}^{*}\right)$ such that $\alpha_{i}(\tilde{h})=0$ for $i \in J$ and $\alpha_{i}(\tilde{h})=2$ for $i \in\{1, \ldots, l\} \backslash J$. Since the map ad $\tilde{e}: \mathfrak{g}(-2 ; \tilde{h}) \longrightarrow \mathfrak{g}(0 ; \tilde{h})$ is bijective, by [7, Cor. 5.2.4], there is $\tilde{f} \in \mathfrak{g}(-2 ; \tilde{h})$ such that $(\tilde{e}, \tilde{h}, \tilde{f})$ is an $\mathfrak{s l}_{2}$-triple in $\mathfrak{g}$.

By construction, $\mathfrak{g}\left(i ; \lambda_{J}\right)=\mathfrak{g}(i ; \tilde{h})$ and $\mathfrak{g}(i ; \tilde{h}) \cap \mathfrak{l}_{I}=\mathfrak{l}_{I}(i ; h)$ for all $i \in \mathbb{Z}$. From this it follows that

$$
[h, \tilde{h}]=0, \quad[\tilde{h}, e]=2 e, \quad \mathfrak{g}(0 ; \tilde{h})=\mathfrak{g}\left(0 ; \lambda_{J}\right)=\mathfrak{l}_{J} .
$$

So the centraliser $\mathfrak{z}(e)$ is $(\operatorname{ad} \tilde{h})$-invariant. From $(2.1)$ we know that nil $\mathfrak{z}(e)$ coincides with $\bigoplus_{i>0} \mathfrak{g}(i ; h) \cap \mathfrak{z}(e)$, hence is also preserved by ad $\tilde{h}$.

Lemma 2.3. The endomorphism ad $\tilde{h}$ acts invertibly on the nilradical nil $\mathfrak{z}(e)$.

Proof. Indeed, it follows from (2) that

$$
\begin{aligned}
\operatorname{nil} \mathfrak{z}(e) \cap \operatorname{Ker} a d \tilde{h} & =\operatorname{nil} \mathfrak{z}(e) \cap \mathfrak{g}(0, \tilde{h})=\operatorname{nil} \mathfrak{z}(e) \cap \mathfrak{l}_{J} \\
& =\operatorname{nil} \mathfrak{z}(e) \cap \mathfrak{l}_{I}(0 ; h) \subseteq \operatorname{nil}\left(\mathfrak{l}_{I}(0 ; h) \cap \mathfrak{z}(e)\right) .
\end{aligned}
$$

Since the Lie algebra $\mathfrak{l}_{I}(0 ; h) \cap \mathfrak{z}(e)$ is reductive, by our discussion in $(2.1)$, the RHS must be zero. The result follows. 
Remark. Using the terminology of the theory of induced orbits [20] one can say that $\tilde{e}$ is a nice correspondent of $e$ in the $G$-orbit $\operatorname{Ind}_{L_{I}, P_{I}}\left(\mathcal{O}_{I}(e)\right)$ where $\mathcal{O}_{I}(e)=\left(\operatorname{Ad} L_{I}\right) e$. 2.4. Let $V$ be a finite dimensional vector space over $\mathbb{C}$. Given an ideal $I$ of the symmetric algebra $S\left(V^{*}\right)$ we denote by gr $I$ the homogeneous ideal of $S\left(V^{*}\right)$ with the property that $g \in \operatorname{gr} I \cap S^{r}\left(V^{*}\right)$ if and only if there is $\tilde{g} \in I$ such that $\tilde{g}-g \in$ $\bigoplus_{i<r} S^{i}\left(V^{*}\right)$. As usual, we identify $S\left(V^{*}\right)$ with the algebra of polynomial functions on $V$. We denote by $\mathcal{Z}\left(\varphi_{1}, \ldots, \varphi_{N}\right)$ the subset in $V$ consisting of all common zeros of $\varphi_{1}, \ldots, \varphi_{N} \in S\left(V^{*}\right)$. Given a Zariski closed set $X \subseteq V$ we let $I_{X}$ be the ideal in $S\left(V^{*}\right)$ consisting of all polynomial functions vanishing on $X$ and define

$$
\mathbb{K}(X):=\left\{v \in V \mid f(v)=0 \quad \forall f \in \operatorname{gr} I_{X}\right\} .
$$

The Zariski closed conical set $\mathbb{K}(X) \subseteq V$ is known as the associated cone to $X$. The proof of our next result will rely on a few elementary properties of the correspondence $X \longmapsto \mathbb{K}(X)$. Our reference here is [18, Chap. 2, (4.2)].

Given an $\mathfrak{s l}_{2}$-triple $(e, h, f)$ in $\mathfrak{g}$ we denote by $\mathcal{S}(h, e)$ the Zariski closure of the orbit $G \cdot(h, e)$ in $\mathfrak{g} \times \mathfrak{g}$. Identify $\mathfrak{g} \times \mathfrak{g}$ with $\mathfrak{g} \oplus \mathfrak{g}$. The next result will play a crucial rôle in our proof of Baranovsky's conjecture.

Proposition 2.4. Let $(e, h, f)$ be an $\mathfrak{s l}_{2}$-triple in $\mathfrak{g}$ with e distinguished. Then there exist pairwise non-conjugate distinguished nilpotent elements $e_{1}, \ldots, e_{k} \in \mathfrak{g}$ such that $\mathbb{K}(\mathcal{S}(h, e))=\mathfrak{C}\left(e_{1}\right) \cup \ldots \cup \mathfrak{C}\left(e_{k}\right)$.

Proof. (1) As $[h, e]=2 e$, any pair $(u, v) \in G \cdot(h, e)$ has the property that $[u, v]=2 v$. Therefore, $\mathcal{S}(h, e) \subseteq\{(x, y) \in \mathfrak{g} \times \mathfrak{g} \mid[x, y]-2 y=0\}$. In view of [18, Chap. 2, Sect. 4.2, Theorem 2(b)] this entails that

$$
\mathbb{K}(\mathcal{S}(h, e)) \subseteq \mathbb{K}(\{(x, y) \in \mathfrak{g} \times \mathfrak{g} \mid[x, y]-2 y=0\}) \subseteq \mathfrak{C}(\mathfrak{g}) .
$$

Let $x_{1}, \ldots, x_{n}$ and $y_{1}, \ldots, y_{n}$ be coordinate functions on $\mathfrak{g} \times\{0\}$ and $\{0\} \times \mathfrak{g}$, respectively. Let $f_{1}, \ldots, f_{l}$ be free homogeneous generators of the invariant algebra $S\left(\mathfrak{g}^{*}\right)^{G}$. It is well-known that $\mathcal{N}=\mathcal{Z}\left(f_{1}, \ldots, f_{l}\right)$. As $\mathcal{S}(h, e) \subseteq(\operatorname{Ad} G) h \times \mathcal{N}$, all polynomial functions $f_{i}\left(x_{1}, \ldots, x_{n}\right)-f_{i}(h)$ and $f_{i}\left(y_{1}, \ldots, y_{n}\right)$ on $\mathfrak{g} \times \mathfrak{g}$ vanish on $\mathcal{S}(h, e)$. This gives $\mathbb{K}(\mathcal{S}(h, e)) \subseteq \mathcal{N} \times \mathcal{N}$. Combining this with (3) one obtains

$$
\mathbb{K}(\mathcal{S}(h, e)) \subseteq \mathfrak{C}^{\text {nil }}(\mathfrak{g})
$$

(2) By the definition of $\mathcal{S}(h, e)$, the orbit morphism

$$
\psi: G \longrightarrow \mathcal{S}(h, e), \quad \psi(g)=((\operatorname{Ad} g) h,(\operatorname{Ad} g) e),
$$

is dominant. The fibre $\psi^{-1}(\psi(1))$ is nothing but the stabiliser of $(h, e)$ in $G$, a closed subgroup of $G$. Its Lie algebra consists of all $x \in \mathfrak{g}$ satisfying $[x, h]=[x, e]=0$, hence coincides with $\mathfrak{z}(e ; 0)$. From $(2.1)$ we know that $\mathfrak{z}(e ; 0)$ is reductive. Because $e$ is distinguished, we have $\mathfrak{z}(e) \subset \mathcal{N}$; so it must be that $\mathfrak{z}(e ; 0)=\{0\}$. Then $\psi^{-1}(\psi(1))$ is finite, implying $\operatorname{dim} \mathcal{S}(h, e)=n=\operatorname{dim} \mathfrak{g}$. Since $\mathcal{S}(h, e)$ is irreducible we now can apply [18, Chap. 2, Sect. 4.2, Theorem 2(c)] to deduce that all irreducible components of $\mathbb{K}(\mathcal{S}(h, e))$ have dimension $n$.

(3) Let $e_{1}, \ldots, e_{r}$ be representatives of the distinguished nilpotent orbits in $\mathfrak{g}$. Let $\mathcal{O}\left(e_{i}\right)$ denote the nilpotent orbit in $\mathfrak{g}$ containing $e_{i}$ where $1 \leq i \leq r$. Since $\pi\left(\mathfrak{C}\left(e_{i}\right)\right)=$ $\overline{\mathcal{O}\left(e_{i}\right)}$ for all $i$, the Zariski closed irreducible sets $\mathfrak{C}\left(e_{1}\right), \ldots, \mathfrak{C}\left(e_{r}\right)$ are pairwise distinct. By our remark in (2.1), for any $e \in \mathcal{N}$ we have $\operatorname{dim} \mathfrak{C}(e) \leq n$ and, moreover, 
$\operatorname{dim} \mathfrak{C}(e)=n$ if and only if $e$ is distinguished. In conjunction with the above discussion this yields that $\mathfrak{C}\left(e_{1}\right), \ldots, \mathfrak{C}\left(e_{r}\right)$ are the irreducible components of highest dimension in $\mathfrak{C}^{\text {nil }}(\mathfrak{g})$. Combining (4) with our final remark in part 2 we now conclude, after renumbering the $e_{i}$ 's if necessary, that $\mathbb{K}(\mathcal{S}(h, e))=\mathfrak{C}\left(e_{1}\right) \cup \ldots \cup \mathfrak{C}\left(e_{k}\right)$ for some $k \leq r$.

2.5. Now all our tools are in place and we are ready for the main result of this section.

Theorem 2.5. Let $\mathfrak{g}$ be a complex semisimple Lie algebra of dimension $n$. All irreducible components of the nilpotent commuting variety $\mathfrak{C}^{\text {nil }}(\mathfrak{g})$ are $n$-dimensional and the number of components equals the number of distinguished nilpotent conjugacy classes in $\mathfrak{g}$. More precisely, $\mathfrak{C}^{\text {nil }}(\mathfrak{g})=\mathfrak{C}\left(e_{1}\right) \cup \ldots \cup \mathfrak{C}\left(e_{r}\right)$ where $e_{1}, \ldots, e_{r}$ are representatives of the distinguished nilpotent orbits in $\mathfrak{g}$.

Proof. Let $e$ be an almost distinguished nilpotent element in $\mathfrak{g}$. In view of Proposition 2.1 it suffices to show that $\mathfrak{C}(e) \subseteq \mathfrak{C}\left(e_{i}\right)$ for some $i \leq r$. Since $\mathfrak{C}(e)=\mathfrak{C}((\operatorname{Ad} g) e)$ for any $g \in G$ no generality will be lost by assuming that $e$ satisfies the conditions of (2.3). We include $e$ into an $\mathfrak{s l}_{2}$-triple $(e, h, f)$ according to the recipe in (2.3) and then consider the corresponding $\mathfrak{s l}_{2}$-triple $(\tilde{e}, \tilde{h}, \tilde{f})$ with $\tilde{e}$ distinguished (see (2.3) for more detail). Thanks to Proposition 2.4 we are then reduced to show that $\mathfrak{C}(e) \subseteq \mathbb{K}(\mathcal{S}(\tilde{h}, \tilde{e}))$

Let $\mathcal{S}=\mathcal{S}(\tilde{h}, \tilde{e})$ and $L=Z_{G}(\tilde{h})$. As $\operatorname{dim} \mathfrak{g}(0 ; \tilde{h})=\operatorname{dim} \mathfrak{g}(2 ; \tilde{h})$ and $\mathfrak{z}(\tilde{e} ; 0)=\{0\}$, the orbit $(\operatorname{Ad} L) \tilde{e}$ is open in $\mathfrak{g}(2 ; \tilde{h})$. Since $\mathcal{S}$ is $L$-stable and Zariski closed, we then have $(\tilde{h}, \mathfrak{g}(2 ; \tilde{h})) \subset \mathcal{S}$. Since $\mathfrak{g}(2 ; \tilde{h}) \cap \mathfrak{l}_{I}=\mathfrak{l}_{I}(2 ; h)$, by $(2.3)$, we obtain $(\tilde{h}, \mathbb{C} e) \subset \mathcal{S}$.

By construction, $\tilde{h} \in$ Lie $T \subseteq \mathfrak{g}(0 ; h)$, see (2.3), while from (2.1) we know that

$$
\text { Lie } R_{u}\left(Z_{G}(e)\right)=\operatorname{nil} \mathfrak{z}(e)=\bigoplus_{i>0} \mathfrak{z}(e ; i) .
$$

As the group $R_{u}\left(Z_{G}(e)\right)$ is unipotent, the orbit $\left(\operatorname{Ad} R_{u}\left(Z_{G}(e)\right)\right) \tilde{h}$ is Zariski closed in $\tilde{h}+\operatorname{nil} \mathfrak{z}(e)$. As $[\operatorname{nil} \mathfrak{z}(e), \tilde{h}]=\operatorname{nil} \mathfrak{z}(e)$, by Lemma 2.3 , it is Zariski open in $\tilde{h}+\operatorname{nil} \mathfrak{z}(e)$ too, and hence is the whole of $\tilde{h}+\operatorname{nil} \mathfrak{z}(e)$. Applying the operators from $R_{u}\left(Z_{G}(e)\right)$ to $(\tilde{h}, \mathbb{C} e) \subset \tilde{S}$ we now derive that $(\tilde{h}+\operatorname{nil} \mathfrak{z}(e), \mathbb{C} e) \subset \mathcal{S}$. But then

$$
\mathbb{K}((\tilde{h}+\operatorname{nil} \mathfrak{z}(e), \mathbb{C} e))=(\operatorname{nil} \mathfrak{z}(e), \mathbb{C} e) \subset \mathbb{K}(\mathcal{S}),
$$

by [18, Chap. 2, Sect. 4.2, Theorem 2(b)]. Since $\mathbb{K}(\mathcal{S})$ is $G$-stable and $e$ is almost distinguished in $\mathfrak{g}$, our discussion in $(2.1)$ yields $G \cdot(\mathcal{N} \cap \mathfrak{z}(e), e) \subseteq \mathbb{K}(\mathcal{S})$. Since $\mathbb{K}(\mathcal{S})$ is $\sigma$-stable, $\mathfrak{C}(e) \subseteq \mathbb{K}(\mathcal{S})$ and our proof is complete.

\section{The modular case}

3.1. In this section we assume that $p=\operatorname{char} k$ is good for $G$. Recall that the derived subgroup $(G, G)$ is simply connected. So there exist simple, simply connected algebraic $k$-groups $G_{1}, \ldots, G_{m}$ such that $(G, G) \cong G_{1} \times \cdots \times G_{m}$. Let $\mathfrak{g}_{i}=$ Lie $G_{i}$ where $1 \leq i \leq m$, and $\mathfrak{g}^{\prime}=\operatorname{Lie}(G, G)$. Then $\mathfrak{g}^{\prime}=\mathfrak{g}_{1} \oplus \cdots \oplus \mathfrak{g}_{m}$, a direct sum of restricted Lie algebras. It is well-known that $\mathcal{N} \subset \mathfrak{g}^{\prime}$; see $[29,(2.3)]$ for example. From this it is immediate that

$$
\mathfrak{C}^{\text {nil }}(\mathfrak{g})=\mathfrak{C}^{\text {nil }}\left(\mathfrak{g}^{\prime}\right) \cong \mathfrak{C}^{\text {nil }}\left(\mathfrak{g}_{1}\right) \times \cdots \times \mathfrak{C}^{\text {nil }}\left(\mathfrak{g}_{m}\right) .
$$

Clearly, a nilpotent element $x=x_{1}+\cdots+x_{m}$ with $x_{i} \in \mathfrak{g}_{i}$ is distinguished in $\mathfrak{g}$ if and only if each $x_{i}$ is distinguished in $\mathfrak{g}_{i}$. Also, $\mathfrak{C}(x) \cong \mathfrak{C}_{G_{1}}\left(x_{1}\right) \times \cdots \times \mathfrak{C}_{G_{m}}\left(x_{m}\right)$ where 
$\mathfrak{C}_{G_{i}}\left(x_{i}\right)$ denotes the Zariski closure of $G_{i} \cdot\left(x_{i}\right.$, Lie $\left.Z_{G_{i}}\left(x_{i}\right)\right)$ in $\mathfrak{g}_{i} \times \mathfrak{g}_{i}$. This observation reduces computing the irreducible components of $\mathfrak{C}^{\text {nil }}(\mathfrak{g})$ to the case where $(G, G)$ is a simple algebraic group.

Until the end of this section we will thus assume that $G$ is either $\operatorname{GL}(n)$ or a simple algebraic group of type different from A. Let $X_{*}(G)$ denote the set of all 1-parameter subgroups $\mu: k^{*} \rightarrow G$. Given $\mu \in X_{*}(G)$ and $i \in \mathbb{Z}$ we denote by $\mathfrak{g}(i ; \mu)$ the subspace of all $x \in \mathfrak{g}$ such that $(\operatorname{Ad} \mu(t)) x=t^{i} x$ for all $t \in k^{*}$. Then $\mathfrak{g}=\bigoplus_{i \in \mathbb{Z}} \mathfrak{g}(i ; \mu)$, that is each $\mu \in X_{*}(G)$ induces a $\mathbb{Z}$-grading of the restricted Lie algebra $\mathfrak{g}$. We denote by $Z(\mu)$ the centraliser of $\mu$ in $G$, a Levi subgroup in $G$.

Let $e$ be a nilpotent element in $\mathfrak{g}$. As in Section 2, we let $\mathfrak{z}(e)$ be the centraliser of $e$ in $\mathfrak{g}, \mathfrak{C}(e)$ be the Zariski closure of $G \cdot(e, \mathcal{N} \cap \mathfrak{z}(e))$, and $\mathcal{O}(e)$ be the $G$-orbit of $e$. Recall from $(2.2)$ that $\mathcal{O}(e)=\mathcal{O}(I, J)$ for some $(I, J) \in \mathcal{P}(\Pi)$. More specifically, there is $g \in G$ such that $(\operatorname{Ad} g) e$ is a Richardson element of $\mathfrak{p}_{I, J}$ contained in $\mathfrak{l}_{I}\left(2 ; \lambda_{I, J}\right)$. Set $\lambda_{e}:=g \lambda_{I, J} g^{-1}$, an element in $X_{*}(G)$. Since $e \in \mathfrak{g}\left(2 ; \lambda_{e}\right)$, the torus $\lambda_{e}\left(k^{*}\right)$ acts on $\mathfrak{z}(e)$. For $i \in \mathbb{Z}$, set $\mathfrak{z}(e ; i):=\mathfrak{z}(e) \cap \mathfrak{g}\left(i ; \lambda_{e}\right)$. According to [16, Sect. 5] and [29, Sect. 2], the group $Z\left(\lambda_{e}\right) \cap Z_{G}(e)$ is reductive, $\operatorname{Lie}\left(Z\left(\lambda_{e}\right) \cap Z_{G}(e)\right)=\mathfrak{z}(e ; 0)$, and

$$
\mathfrak{z}(e)=\operatorname{Lie} Z_{G}(e)=\bigoplus_{i \geq 0} \mathfrak{z}(e ; i), \quad \text { Lie } R_{u}\left(Z_{G}(e)\right)=\bigoplus_{i>0} \mathfrak{z}(\mathfrak{g}, i) .
$$

This implies that the nilpotent variety $\mathcal{N}(\mathfrak{z}(e ; 0))=\mathcal{N} \cap \mathfrak{z}(e ; 0)$ of $\mathfrak{z}(e ; 0)$ is irreducible. Hence the varieties $\mathcal{N} \cap \mathfrak{z}(e) \cong \mathcal{N}(\mathfrak{z}(e ; 0)) \times \bigoplus_{i>0} \mathfrak{z}(e ; i)$ and $\mathfrak{C}(e)$ are irreducible, too. The morphism

$$
\xi: G \times(\mathcal{N} \cap \mathfrak{z}(e)) \longrightarrow \mathfrak{C}(e), \quad \xi(g, x)=((\operatorname{Ad} g) e,(\operatorname{Ad} g) x)
$$

is dominant and the fibre $\xi^{-1}(\xi(g, x))$ is nothing but the set of all pairs $\left(a,\left(\operatorname{Ad}^{-1} g\right) x\right)$ with $g^{-1} a \in Z_{G}(e)$. It follows that $\xi^{-1}(\xi(g, x)) \cong Z_{G}(e)$ as varieties. The theorem on the dimension of the fibres of a morphism combined with our earlier remarks yields

$$
\begin{aligned}
\operatorname{dim} \mathfrak{C}(e) & =\operatorname{dim} G+\operatorname{dim} \mathcal{N} \cap \mathfrak{z}(e)-\operatorname{dim} Z_{G}(e) \\
& =\operatorname{dim} G+\operatorname{dim} \mathcal{N} \cap \mathfrak{z}(e)-\operatorname{dim} \mathfrak{z}(e) \\
& =\operatorname{dim} G-\operatorname{rk}\left(Z\left(\lambda_{e}\right) \cap Z_{G}(e)\right) \\
& =\operatorname{dim}(G, G)-\operatorname{rk}\left(Z\left(\lambda_{e}\right) \cap Z_{(G, G)}(e)\right) .
\end{aligned}
$$

As a consequence, $\operatorname{dim} \mathfrak{C}(e) \leq \operatorname{dim}(G, G)$ for any $e \in \mathcal{N}$. As $Z(G) \subset Z\left(\lambda_{e}\right)$ and $Z_{G}(e)=Z(G) \cdot Z_{(G, G)}(e)$ we have that $Z\left(\lambda_{e}\right) \cap Z_{G}(e)=Z(G) \cdot Z\left(\lambda_{e}\right) \cap Z_{(G, G)}(e)$. Thus $\operatorname{dim} \mathfrak{C}(e)=\operatorname{dim}(G, G)$ if and only if the reductive group $Z\left(\lambda_{e}\right) \cap Z_{(G, G)}(e)$ is finite, i.e. if and only if $e$ is distinguished in $\mathfrak{g}$.

Lemma 3.1. Let $e \in \mathcal{N}$ be such that $\mathcal{N} \cap \mathfrak{z}(e) \subset \overline{\mathcal{O}(e)}$. Then every nilpotent element of $\mathfrak{z}(e ; 0)$ is contained in $\mathfrak{z}(e ; 0) \cap\left[e, \mathfrak{g}\left(-2 ; \lambda_{e}\right)\right]$.

Proof. Set $H=Z_{G}\left(\lambda_{e}\right) \cap Z_{G}(e)$. Since $H$ is reductive any nilpotent $e_{0} \in$ Lie $H=$ $\mathfrak{z}(e ; 0)$ satisfies $0 \in \overline{(\operatorname{Ad} H) e_{0}}$; see [3] for example. It follows that $\overline{(\operatorname{Ad} H)\left(e+e_{0}\right)}=$ $e+\overline{(\operatorname{Ad} H) e_{0}}$ contains $e$. As a result, $\mathcal{O}(e) \subseteq \overline{\mathcal{O}\left(e+e_{0}\right)}$. Since $e+e_{0} \in \mathcal{N} \cap \mathfrak{z}(e)$, the assumption on $e$ implies that $\overline{\mathcal{O}(e)}=\overline{\mathcal{O}\left(e+e_{0}\right)}$ and thus $e+e_{0} \in \mathcal{O}(e)$. Since $k e_{0} \subset \mathcal{N} \cap \mathfrak{z}(e ; 0)$ this yields $e+k e_{0} \subset \mathcal{O}(e)$ forcing $e_{0} \in T_{e} \mathcal{O}(e)$.

Our assumption on $G$ implies that the orbit map $g \longmapsto(\operatorname{Ad} g) e$ is separable; see [35, Chap. I, Sect. 5]. Therefore, $T_{e} \mathcal{O}(e)=[\mathfrak{g}, e]$ and $e_{0} \in[e, \mathfrak{g}] \cap \mathfrak{z}(e ; 0)=$ $\mathfrak{z}(e ; 0) \cap\left[e, \mathfrak{g}\left(-2 ; \lambda_{e}\right)\right]$ as claimed. 
Remark. 1) The proof of Lemma 3.1 shows that the main point in step (3) of the proof of Theorem 2.1 (the conjugacy of $e$ and $e+e_{0}$ ) generalises to our present case. Steps (1) and (2) of the proof are also valid in good characteristic.

2) It follows from Theorem 5.4.8 and Proposition 5.5.2 in [7] that the linear map $(\operatorname{ad} e)^{2}: \mathfrak{g}\left(-2 ; \lambda_{e}\right) \longrightarrow \mathfrak{g}\left(2 ; \lambda_{e}\right)$ is bijective if $p=$ char $k$ is either 0 or sufficiently large. As a consequence, $\mathfrak{z}(e ; 0) \cap\left[e, \mathfrak{g}\left(-2 ; \lambda_{e}\right)\right]=\{0\}$ under these assumptions on $p$. Thus the proof of Lemma 3.1 also shows that for $p=0$ and for $p \gg 0$ the conjugacy of $e$ and $e+e_{0}$ with $e_{0} \in \mathfrak{z}(e ; 0)$ always implies that $e_{0}=0$. In characteristic 0 , this argument can be used as an alternative to step (4) of the proof of Theorem 2.1.

3) In small characteristic, it is not unusual that $e$ is $G$-conjugate to $e+e_{0}$ for some nonzero nilpotent $e_{0} \in \mathfrak{z}(e ; 0)$. For example, let $V$ be a vector space over $k$ with basis $v_{1}, \ldots, v_{p}, w_{1}, \ldots, w_{p}$, and let $\mathfrak{g}=\mathfrak{g l}(V)$. Put $v_{i}=w_{i}=0$ for $i>p$ and let $e$ be the nilpotent element in $\mathfrak{g}=\mathfrak{g l}(V)$ such that $e\left(v_{i}\right)=v_{i+1}$ and $e\left(w_{i}\right)=w_{i+1}$ for all $i$. Let $e_{0}$ be the nilpotent element in $\mathfrak{g}$ such that $e_{0}\left(v_{i}\right)=w_{i}$ and $e_{0}\left(w_{i}\right)=0$ for all $i$. Clearly, $\left[e, e_{0}\right]=0$ and $e^{p}=e_{0}^{p}=0$. It follows that $\left(e+e_{0}\right)^{p}=e^{p}+e_{0}^{p}=0$. Straightforward computation shows that $\operatorname{Ker}\left(e+e_{0}\right)$ is 2-dimensional. From this it is immediate that $e$ and $e+e_{0}$ are conjugate under $G=\mathrm{GL}(V)$, while from the definition of $\lambda_{e}$ in [29, Sect. 2] and the explicit description of $\lambda_{e}$ in [35, Chap. IV, (1.6)] it follows that $e_{0} \in \mathfrak{z}(e ; 0)$.

3.2. We say that a nilpotent element $e \in \mathfrak{g}$ is almost distinguished if the connected component of $Z_{G}(e)$ is a solvable group. For $p=0$ this is consistent with our definition in $(2.1)$.

Proposition 3.2. Let $G$ be as above and let $e \in \mathcal{N}$ be such that $\mathcal{N} \cap \mathfrak{z}(e) \subset \overline{\mathcal{O}(e)}$. Then e is almost distinguished in $\mathfrak{g}$.

Proof. Suppose $e$ is not almost distinguished in $\mathfrak{g}$ (then $e$ is not distinguished in $\mathfrak{g}$ either). Since the connected component of $Z_{G}(e) / R_{u}\left(Z_{G}(e)\right) \cong Z\left(\lambda_{e}\right) \cap Z_{G}(e)$ is not a torus, by our assumption on $e$, and since $\mathfrak{z}(e ; 0)=\operatorname{Lie}\left(Z\left(\lambda_{e}\right) \cap Z_{G}(e)\right)$, by [16, Sect. 5] and [29, Sect. 2], it must be that $\mathcal{N}(\mathfrak{z}(e ; 0)) \neq\{0\}$. Then $\mathfrak{z}(e ; 0) \cap\left[e, \mathfrak{g}\left(-2 ; \lambda_{e}\right)\right] \neq\{0\}$, by Lemma 3.1. So the linear map $(\operatorname{ad} e)^{2}: \mathfrak{g}\left(-2 ; \lambda_{e}\right) \longrightarrow \mathfrak{g}\left(2 ; \lambda_{e}\right)$ is not bijective. As mentioned in the remark at the end of (3.1), this can only happen when $p$ is small.

Our further arguments will rely on various case-by-case considerations most of which will be presented in the Appendix (one can skip these technical details in the first reading). However, the type A case will be considered in the main body of the paper. It is of particular interest to us since we have in mind an application to the punctual Hilbert scheme $\mathcal{H}_{n}$ over $k$.

(1) Suppose $G=\operatorname{GL}(V)$. Let $V=V_{1} \oplus \cdots \oplus V_{s}$ be a decomposition of $V$ into a direct sum of indecomposable $k e$-modules such that $\operatorname{dim} V_{1} \geq \cdots \geq \operatorname{dim} V_{s}$. The partition of $\operatorname{dim} V$ associated with $e$ is nothing but $\left(\operatorname{dim} V_{1}, \operatorname{dim} V_{2}, \ldots, \operatorname{dim} V_{s}\right)$.

Let $I=\left\{i \in\{1, \ldots, s-1\} \mid \operatorname{dim} V_{i}=\operatorname{dim} V_{i+1}\right\}$. Suppose $I \neq \emptyset$ and let $j$ be the smallest integer in $I$. Let $d=\operatorname{dim} V_{j}=\operatorname{dim} V_{j+1}$ and $M=V_{j} \oplus V_{j+1}$. Let $v_{1}, \ldots, v_{d}$ and $w_{1}, \ldots, w_{d}$ be bases of $V_{j}$ and $V_{j+1}$ such that $e\left(v_{d}\right)=e\left(w_{d}\right)=0$ and $e\left(v_{i}\right)=v_{i+1}$, $e\left(w_{i}\right)=w_{i+1}$ for $i<d$. There is $z \in \mathfrak{g l}(M)$ such that $z\left(w_{d}\right)=0, z\left(v_{i}\right)=w_{i}$ for $1 \leq i \leq d$ and $z\left(w_{i}\right)=v_{i+1}$ for $1 \leq i<d$. For $i>d$, put $v_{i}=w_{i}=0$. Then $z e\left(v_{i}\right)=w_{i+1}=e z\left(v_{i}\right)$ and $z e\left(w_{i}\right)=v_{i+2}=e z\left(w_{i}\right)$ for all $i$. In other words, the 
endomorphisms $e_{\mid M}$ and $z$ commute. Note that $z$ acts on $M$ as a nilpotent Jordan block of order $2 d$. There is $\hat{z} \in \mathfrak{g l}(V)$ such that $\hat{z}_{\mid M}=z$ and $\hat{z}_{\mid V_{i}}=e_{\mid V_{i}}$ for all $i \notin\{j, j+1\}$. By construction, $\hat{z} \in \mathcal{N} \cap \mathfrak{z}(e)$ and the partition of $\operatorname{dim} V$ associated with $\hat{z}$ is not dominated by that of $e$. Applying [10] we now deduce that $\hat{z} \notin \overline{\operatorname{GL}(V) e}$. Since this contradicts our assumption on $e$ it must be that $I=\emptyset$.

As a result, all parts of the partition of $\operatorname{dim} V$ associated with $e$ are distinct. But then $e$ is almost distinguished in $\mathfrak{g}$, by [35, Chap. IV, Cor. 1.8(i)]. So the proposition holds in the present case. The rest of the proof can be found in the Appendix.

3.3. Proposition 3.2 enables us now to establish a modular version of Proposition 2.1.

Proposition 3.3. Under the present assumption on $G$, each irreducible component of $\mathfrak{C}^{\text {nil }}(\mathfrak{g})$ is of the form $\mathfrak{C}(e)$ where $e$ is almost distinguished in $\mathfrak{g}$.

Proof. By [13], the nilpotent orbits in reductive Lie algebras are finite in number regardless of $p$. Therefore, our arguments in parts 1 and 2 of the proof of Proposition 2.1 apply to any reductive Lie algebra. They show that each irreducible component of $\mathfrak{C}^{\text {nil }}(\mathfrak{g})$ is of the form $\mathfrak{C}(e)$ where $e \in \mathcal{N}$ is such that $\mathcal{N} \cap \mathfrak{z}(e) \subset \overline{\mathcal{O}(e)}$. According to Proposition 3.2, each such $e$ is almost distinguished in $\mathfrak{g}$.

3.4. Recall that all our observations in (2.2) are valid in the present setting. With the notation of $(2.2)$, we pick $(I, J)$ in $\mathcal{P}(\Pi)$ and let $e \in \mathfrak{l}_{I}\left(2 ; \lambda_{I, J}\right)$ be such that the orbit $\left(\operatorname{Ad} P_{I, J}\right) e$ is open in $\mathfrak{u}_{I, J}$. By Lemma 2.2, $P_{J}$ is a distinguished parabolic subgroup of $G$; so $\lambda_{J} \in X_{*}(T)$ is well-defined. By [7, Prop. 5.8.5], $\mathfrak{p}_{J}=\bigoplus_{i>0} \mathfrak{g}\left(2 i ; \lambda_{J}\right)$ and the subspace $\mathfrak{g}\left(2 ; \lambda_{J}\right)$ contains a Richardson element of $\mathfrak{p}_{J}$. As in $(2 . \overline{3})$, we pick such an element and call it $\tilde{e}$. It follows from the definition of $\lambda_{J}$ that

$$
\mathfrak{g}\left(i ; \lambda_{J}\right) \cap \mathfrak{l}_{I}=\mathfrak{l}_{I}\left(i ; \lambda_{I, J}\right) \quad(\forall i \in \mathbb{Z}) .
$$

In particular, $e \in \mathfrak{g}\left(2 ; \lambda_{J}\right)$ so that $\operatorname{Int} \lambda_{J}\left(k^{*}\right)$ acts on $Z_{G}(e)$. As a result, $\operatorname{Ad} \lambda_{J}\left(k^{*}\right)$ acts on Lie $R_{u}\left(Z_{G}(e)\right)$ and on each weight space $\mathfrak{g}\left(i ; \lambda_{I, J}\right)$ of $\lambda_{I, J}\left(k^{*}\right)$.

Lemma 3.4. The torus $\operatorname{Ad} \lambda_{J}\left(k^{*}\right)$ has no zero weight on Lie $R_{u}\left(Z_{G}(e)\right)$.

Proof. It follows from [16, Sect. 5] and [29, Sect. 2] that

$$
\text { Lie } R_{u}\left(Z_{G}(e)\right)=\bigoplus_{i>0} \mathfrak{g}\left(i ; \lambda_{I, J}\right) \cap \mathfrak{z}(e),
$$

while from the definition of $\lambda_{J}$ it is immediate that $\mathfrak{g}\left(0 ; \lambda_{J}\right)=\mathfrak{l}_{J} \subseteq \mathfrak{l}_{I}$. Then

$$
\text { Lie } \begin{aligned}
R_{u}\left(Z_{G}(e)\right) \cap \mathfrak{g}\left(0 ; \lambda_{J}\right) & =\bigoplus_{i>0} \mathfrak{g}\left(i ; \lambda_{I, J}\right) \cap \mathfrak{l}_{J} \cap \mathfrak{z}(e) \\
& \subseteq \bigoplus_{i>0} \mathfrak{l}_{I}\left(i ; \lambda_{I, J}\right) \cap \mathfrak{l}_{J}=\{0\},
\end{aligned}
$$

in view of $(5)$ and the equality $\mathfrak{l}_{J}=\mathfrak{l}_{I}\left(0 ; \lambda_{I, J}\right)$. The result follows.

3.5. Given a linear algebraic group $H$ we denote by $\mathcal{U}(H)$ the unipotent variety of $H$ and put $\mathcal{U}=\mathcal{U}(G)$. Note that $\mathcal{U} \subset(G, G)$. Since the $\mathfrak{s l}_{2}$-theory has its limitations, we have to modify our constructions in (2.4): associated cones do not work for $p$ small. This goal will be achieved in (3.6) where we introduce a group analogue of $\mathbb{K}(\mathcal{S}(\tilde{h}, \tilde{e}))$. The latter will be linked with $\mathfrak{C}^{\text {nil }}(\mathfrak{g})$ by means of a $G$-equivariant isomorphism between $\mathcal{U}$ and $\mathcal{N}$.

In fact, we need the $G$-equivariant isomorphism between $\mathcal{U}$ and $\mathcal{N}$ introduced in [3]. This isomorphism, call it $\eta$, is defined in loc. cit. as follows: If $G=\operatorname{GL}(V)$, one just puts $\eta(u)=u-1$ for all $u \in \mathcal{U}$. If $G$ is not of type A, then $\mathfrak{g}$ is a simple Lie algebra. 
In this case one picks a finite dimensional rational representation $\rho: G \rightarrow \operatorname{GL}(W)$ with ker $\rho \subseteq Z(G)$ such that the trace form $(X, Y) \longmapsto \operatorname{tr}(\mathrm{d} \rho)_{e}(X) \circ(\mathrm{d} \rho)_{e}(Y)$ on $\mathfrak{g}$ is nondegenerate; see [35, Chap. I, Lemma 5.3]. One then identifies $\mathfrak{g}$ with its image $(\mathrm{d} \rho)(\mathfrak{g})$. By the choice of $\rho$, there is a subspace $M \subset \mathfrak{g l}(W)$ such that $\mathfrak{g l}(W)=$ $\mathfrak{g} \oplus M$ and $[\mathfrak{g}, M] \subseteq M$. Specifically, $M$ is the orthogonal complement of $\mathfrak{g}$ with respect to the trace form on $\mathfrak{g l}(W)$. Finally, one takes for $\eta$ the restriction of $\operatorname{pr}_{1} \circ \rho$ to $\mathcal{U}$ where $\operatorname{pr}_{1}: \mathfrak{g l}(W) \rightarrow \mathfrak{g}$ is the first projection. According to [3, Cor. 9.3.4], $\eta: \mathcal{U} \stackrel{\sim}{\longrightarrow} \mathcal{N}$ is a $G$-equivariant isomorphism of algebraic varieties. Hence so is the $\operatorname{map} \eta \times \operatorname{id}_{\mathcal{N}}: \mathcal{U} \times \mathcal{N} \longrightarrow \mathcal{N} \times \mathcal{N}$.

We now define $\mathfrak{X}:=\{(u, x) \in \mathcal{U} \times \mathcal{N} \mid(\operatorname{Ad} u) x=x\}$, a closed subset in $\mathcal{U} \times \mathcal{N}$, and denote by $\tilde{\eta}$ the restriction of $\eta \times \mathrm{id}_{\mathcal{N}}$ to $\mathfrak{X}$.

Lemma 3.5. The map $\tilde{\eta}$ is a $G$-equivariant isomorphism between $\mathfrak{X}$ and $\mathfrak{C}^{\text {nil }}(\mathfrak{g})$. For any $e \in \mathcal{N}$ it maps $\left(R_{u}\left(Z_{G}(e)\right), e\right)$ onto $\left(\right.$ Lie $\left.R_{u}\left(Z_{G}(e)\right), e\right)$.

Proof. Let $(u, e) \in \mathfrak{X}$. Then $(\operatorname{Ad} u) e=e$ forcing $\rho(u) e \rho(u)^{-1}=e$ (recall that we identify $\mathfrak{g}$ with $(\mathrm{d} \rho)(\mathfrak{g}))$. Thus $\rho(u)$ commutes with $e$. Write $\rho(u)=x+m$ with $x \in \mathfrak{g}$ and $m \in M$. Then $x=\eta(u) \in \mathcal{N}$ and $0=[e, \rho(u)]=[e, x]+[e, m]$. Hence $[e, \eta(u)]=0$. This shows that $\tilde{\eta}(\mathfrak{X}) \subseteq \mathfrak{C}^{\text {nil }}(\mathfrak{g})$.

As a consequence, $\tilde{\eta}$ sends $\left(\mathcal{U} \cap \bar{Z}_{G}(e), e\right)$ to $(\mathcal{N} \cap \mathfrak{z}(e), e)$. From [16, Sect. 5] and $\left[29\right.$, Sect. 2] we know that $\mathcal{U} \cap Z_{G}(e)$ is isomorphic to $\mathcal{U}\left(Z\left(\lambda_{e}\right) \cap Z_{G}(e)\right) \times R_{u}\left(Z_{G}(e)\right)$. Our discussion in (3.1) shows that

$$
\mathcal{N} \cap \mathfrak{z}(e) \cong \mathcal{N}\left(\text { Lie } Z\left(\lambda_{e}\right) \cap Z_{G}(e)\right) \times \text { Lie } R_{u}\left(Z_{G}(e)\right)
$$

is an irreducible variety. Since $Z\left(\lambda_{e}\right) \cap Z_{G}(e)$ is a reductive group, the varieties $\mathcal{N}\left(\right.$ Lie $\left.Z\left(\lambda_{e}\right) \cap Z_{G}(e)\right)$ and $\mathcal{U}\left(Z\left(\lambda_{e}\right) \cap Z_{G}(e)\right)$ have the same dimension. This implies that $\operatorname{dim} \mathcal{U} \cap Z_{G}(e)=\operatorname{dim} \mathcal{N} \cap \mathfrak{z}(e)$.

Since $\eta \times \mathrm{id}_{\mathcal{N}}$ is an isomorphism of varieties, it maps the closed set $\left(\mathcal{U} \cap Z_{G}(e), e\right)$ onto a closed subset of $(\mathcal{N} \cap \mathfrak{z}(e), e)$ of the same dimension. The irreducibility of $(\mathcal{N} \cap \mathfrak{z}(e), e)$ now yields that $\tilde{\eta}$ maps $\left(\mathcal{U} \cap Z_{G}(e), e\right)$ onto $(\mathcal{N} \cap \mathfrak{z}(e), e)$. Since this holds for any $e \in \mathcal{N}$, it must be that $\tilde{\eta}(\mathfrak{X})=\mathfrak{C}^{\text {nil }}(\mathfrak{g})$. But then $\tilde{\eta}: \mathfrak{X} \longrightarrow \mathfrak{C}^{\text {nil }}(\mathfrak{g})$ is a $G$-equivariant isomorphism of varieties.

Let $P\left(\lambda_{e}\right)$ be the parabolic subgroup of $G$ with Lie algebra $\mathfrak{p}\left(\lambda_{e}\right):=\bigoplus_{i>0} \mathfrak{g}\left(i ; \lambda_{e}\right)$. It follows from [21, Lemma 28] that $\eta$ maps $R_{u}\left(P\left(\lambda_{e}\right)\right)$ onto Lie $R_{u}\left(P\left(\bar{\lambda}_{e}\right)\right)$. Note that Lie $R_{u}\left(P\left(\lambda_{e}\right)\right)=\bigoplus_{i>0} \mathfrak{g}\left(i ; \lambda_{e}\right)$. Since $\tilde{\eta}$ maps $\left(\mathcal{U} \cap Z_{G}(e), e\right)$ onto $(\mathcal{N} \cap \mathfrak{z}(e), e)$, it maps $\left(R_{u}\left(P\left(\lambda_{e}\right)\right) \cap Z_{G}(e), e\right)$ onto (Lie $\left.R_{u}\left(P\left(\lambda_{e}\right)\right) \cap \mathfrak{z}(e), e\right)$. Combined with [16, Sect. 5] or [29, Sect. 2] this yields that $\tilde{\eta}$ maps $\left(R_{u}\left(Z_{G}(e)\right), e\right)$ onto $\left(\right.$ Lie $\left.R_{u}\left(Z_{G}(e)\right), e\right)$ as desired.

Remark. Let $\mathfrak{C}^{\text {unip }}(G):=\{(x, y) \in \mathcal{U} \times \mathcal{U} \mid x y=y x\}$ be the unipotent commuting variety of $G$. Since both $\rho(\mathfrak{g})$ and $M$ are stable under all Int $\rho(g)$ with $g \in G$, the argument used in the proof of Lemma 3.5 also shows the map id $\operatorname{U}_{\mathcal{U}} \times \eta$ induces a $G$ equivariant isomorphism between $\mathfrak{C}^{\text {unip }}(G)$ and $\mathfrak{X}$. As a result, the varieties $\mathfrak{C}^{\text {unip }}(G)$ and $\mathfrak{C}^{\text {nil }}(\mathfrak{g})$ are $G$-equivariantly isomorphic.

3.6. The group $G$ acts on $G \times \mathcal{N}$ via $g \cdot(x, n)=((\operatorname{Int} g) x,(\operatorname{Ad} g) n)$ fot all $g, x \in G$ and $n \in \mathcal{N}$. Given a distinguished nilpotent element $e \in \mathfrak{g}$ we denote by $\mathcal{Y}\left(\lambda_{e}, e\right)$ the Zariski closure of $G \cdot\left(\lambda_{e}\left(k^{*}\right), e\right)$ in $G \times \mathcal{N}$. By the definition of $\mathcal{Y}\left(\lambda_{e}, e\right)$, the morphism

$$
\nu: G \times k^{*} \longrightarrow \mathcal{Y}\left(\lambda_{e}, e\right), \quad(g, t) \longmapsto\left(g \lambda_{e}(t) g^{-1},(\operatorname{Ad} g) e\right),
$$


is dominant. It follows that $\mathcal{Y}\left(\lambda_{e}, e\right)$ is an irreducible variety. Since $k$ is infinite, there is $t_{0} \in k^{*}$ such that $Z_{G}\left(\lambda_{e}\right)=Z_{G}\left(\lambda_{e}\left(t_{0}\right)\right)$. The fibre $\nu^{-1}\left(\nu\left(1, t_{0}\right)\right)=\nu^{-1}\left(\lambda_{e}\left(t_{0}\right), e\right)$ is nothing but the set of all pairs $(g, t)$ with $g \in Z_{G}(e)$ and $g^{-1} \lambda_{e}\left(t_{0}\right) g=\lambda_{e}(t)$. Since $\lambda_{e}$ is optimal for $e$ the centraliser $Z_{G}(e)$ is contained in $P\left(\lambda_{e}\right)$; see $[29,(2.3)]$ for more detail. From this it follows that $\lambda_{e}(t)=g^{-1} \lambda_{e}\left(t_{0}\right) g=\lambda_{e}\left(t_{0}\right) u$ for some unipotent $u \in G$. Since $\lambda_{e}\left(t_{0}\right)^{-1} \lambda_{e}(t)$ is semisimple, it must be that $u=1$. Consequently, $\nu^{-1}\left(\nu\left(1, t_{0}\right)\right)$ coincides with $\operatorname{Stab}_{G}\left(\lambda_{e}\left(t_{0}\right), e\right)$, the stabiliser of $\left(\lambda_{e}\left(t_{0}\right), e\right)$ in $G$. In view of $[4$, Chap. III, $(9.1),(9,4)]$ and the choice of $t_{0}$, we have

$$
\text { Lie }\left(\operatorname{Stab}_{G}\left(\lambda_{e}\left(t_{0}\right), e\right)\right) \subseteq \mathfrak{z}(e) \cap \text { Lie } Z_{G}\left(\lambda_{e}\left(t_{0}\right)\right)=\mathfrak{z}(e) \cap \mathfrak{g}\left(0 ; \lambda_{e}\right)=\mathfrak{z}(e ; 0) .
$$

Since $e$ is distinguished, the reductive part of $Z_{G}(e)^{\circ}=\left(Z(G) \cdot Z_{(G, G)}(e)\right)^{\circ}$ coincides with $Z(G)^{\circ}$, the connected centre of $G$. Combining this with [16, Sect. 5] and $[29$ Sect. 2] one derives that $\mathfrak{z}(e ; 0)$ coincides with $\mathfrak{z}(\mathfrak{g})$, the centre of $\mathfrak{g}$. By our assumption on $G$, we have $\mathfrak{z}(\mathfrak{g})=\{0\}$ unless $G$ is of type A, in which case $\operatorname{dim} \mathfrak{z}(\mathfrak{g})=\operatorname{dim} Z(G)^{\circ}=$ 1. This implies that $\left(\operatorname{Stab}_{G}\left(\lambda_{e}\left(t_{0}\right), e\right)\right)^{\circ}=Z(G)^{\circ}$. Consequently, $\operatorname{dim} \nu^{-1}\left(\nu\left(1, t_{0}\right)\right)=$ $\operatorname{dim} Z(G)^{\circ}$. On the other hand, it is easy to see that $\operatorname{dim} \nu^{-1}(\nu(g, t)) \geq \operatorname{dim} Z(G)^{\circ}$ for all $(g, t) \in G \times k^{*}$. Applying the theorem on the dimensions of the fibres of a morphism we now get

$$
\operatorname{dim} \mathcal{Y}\left(\lambda_{e}, e\right)=\operatorname{dim} G-\operatorname{dim} Z(G)^{\circ}+1=\operatorname{dim}(G, G)+1 .
$$

Now let $l=\operatorname{rk}(G, G)$ and let $\rho_{1}, \ldots, \rho_{l}$ be the fundamental representations of $G$ (when $G=G L(V)$, we take as $\rho_{i}$ the $i$ th exterior power of the vector representation of $G$ ). For $1 \leq i \leq l$, define the regular invariant function $\chi_{i}$ on $G$ by setting $\chi_{i}(g)=\operatorname{tr} \rho_{i}(g)$ for all $g \in G$. Define $\chi_{0} \in k[G]^{G}$ by setting, for all $g \in G$,

$$
\chi_{0}(g)= \begin{cases}\operatorname{det} g & \text { when } G \text { is of type A, } \\ 1 & \text { otherwise. }\end{cases}
$$

Define the morphism $F: \mathcal{Y}\left(\lambda_{e}, e\right) \longrightarrow \mathbb{A}^{l+1}$ by letting

$$
F(g, n)=\left(\chi_{0}(g), \chi_{1}(g), \ldots, \chi_{l}(g)\right) \quad \forall(g, n) \in \mathcal{Y}\left(\lambda_{e}, e\right),
$$

and denote by $\mathcal{Y}_{0}\left(\lambda_{e}, e\right)$ the fibre $F^{-1}(F(1,0))$.

Proposition 3.6. $\mathcal{Y}_{0}\left(\lambda_{e}, e\right)$ is a closed subset in $\mathfrak{X}$ and all irreducible components of $\mathcal{Y}_{0}\left(\lambda_{e}, e\right)$ have dimension equal to $\operatorname{dim}(G, G)$.

Proof. (1) First note that $(G, G)=\left\{g \in G \mid \chi_{0}(g)=1\right\}$. Together with [36, (3.4), Cor. 4] this implies that $\mathcal{U}=\left\{g \in G \mid \chi_{i}(g)=\chi_{i}(1)\right.$ for all $\left.i\right\}$ (one should take into account that the group $(G, G)$ is simply connected). It follows that $\mathcal{Y}_{0}\left(\lambda_{e}, e\right) \subseteq \mathcal{U} \times \mathcal{N}$. On the other hand, the set $\mathcal{R}:=\{(g, x) \in G \times \mathcal{N} \mid(\operatorname{Ad} g) x \in k x\}$ is Zariski closed and $G$-stable in $G \times \mathcal{N}$. As $\left(\lambda_{e}\left(k^{*}\right), e\right) \subset \mathcal{R}$, this yields $\mathcal{Y}\left(\lambda_{e}, e\right) \subseteq \mathcal{R}$. If $u \in \mathcal{U}$, then 1 is the only eigenvalue of $\operatorname{Ad} u$. Hence $(\mathcal{U} \times \mathcal{N}) \cap \mathcal{R} \subseteq \mathfrak{X}$ forcing

$$
\mathcal{Y}_{0}\left(\lambda_{e}, e\right) \subseteq(\mathcal{U} \times \mathcal{N}) \cap \mathcal{R} \subseteq \mathfrak{X}
$$

(2) Let $\mathcal{C}$ denote the Zariski closure of $\left\{F\left(\lambda_{e}(t), 0\right) \mid t \in k^{*}\right\}$ in $\mathbb{A}^{l+1}$. Since $\lambda_{e}: k^{*} \rightarrow G$ is a nontrivial homomorphism, $\mathcal{C}$ is an affine curve. Since all $\chi_{i}$ are $G$-invariant, $F$ maps $\mathcal{Y}\left(\lambda_{e}, e\right)$ onto a Zariski open subset of $\mathcal{C}$, say $\mathcal{C}_{0}$. Clearly, $\mathcal{C}_{0}$ is a curve on its own and the morphism $F: \mathcal{Y}\left(\lambda_{e}, e\right) \longrightarrow \mathcal{C}_{0}$ is surjective. As $\mathcal{Y}\left(\lambda_{e}, e\right)$ is irreducible, we now combine [32, Chap. I, $\S 6$, Theorem 7] with (6) to deduce that all irreducible 
components of $F^{-1}(F(1,0))=\mathcal{Y}_{0}\left(\lambda_{e}, e\right)$ have dimension equal to $\operatorname{dim}(G, G)$. This completes the proof.

3.7. Now we are ready for the main result of this section.

Theorem 3.7. Let $G$ be a connected reductive group over $k$ and assume that $p=$ char $k$ is a good prime for $G$. Let $\mathfrak{g}=$ Lie $G$ and $n=\operatorname{dim}(G, G)$. Then all irreducible components of $\mathfrak{C}^{\text {nil }}(\mathfrak{g})$ are $n$-dimensional and their number equals the number of distinguished nilpotent conjugacy classes in $\mathfrak{g}$. Moreover, each irreducible component of $\mathfrak{C}^{\text {nil }}(\mathfrak{g})$ has the form $\mathfrak{C}(e)$ for some distinguished nilpotent element $e \in \mathfrak{g}$.

Proof. (1) As explained in (3.1), it can be assumed in proving this theorem that $G$ is either $\mathrm{GL}(n)$ or a simple algebraic group of type different from A. Let $e$ be an almost distinguished nilpotent element in $\mathfrak{g}$. Thanks to Proposition 3.3 it suffices to show that $\mathfrak{C}(e) \subseteq \mathfrak{C}\left(e^{\prime}\right)$ for some distinguished $e^{\prime} \in \mathcal{N}$. Since $\mathfrak{C}(e)=\mathfrak{C}((\operatorname{Ad} g) e)$ for any $g \in G$ we may assume that $e$ is as in (3.4); in particular, $e$ is Richardson in $\mathfrak{u}_{I, J}$ for some $(I, J) \in \mathcal{P}(\Pi)$. We now pick a distinguished $\tilde{e} \in \mathcal{N}$ following the procedure described in (3.4) and take as $\lambda_{\tilde{e}}$ the 1-parameter subgroup $\lambda_{J}$.

(2) We claim that $\left(\lambda_{\tilde{e}}\left(k^{*}\right) R_{u}\left(Z_{G}(e)\right), e\right) \subset \mathcal{Y}\left(\lambda_{\tilde{e}}, \tilde{e}\right)$. To that end we let $b_{1}, \ldots, b_{m}$ be the weights of $\left(\operatorname{Ad} \lambda_{\tilde{e}}\right)\left(k^{*}\right)$ on Lie $R_{u}\left(Z_{G}(e)\right)$. By Lemma 3.4, none of them is zero. It follows that $k_{\text {reg }}^{*}:=\left\{t \in k^{*} \mid b_{i}\left(\operatorname{Ad} \lambda_{\tilde{e}}(t)\right) \neq 1\right.$ for all $\left.i\right\}$ is a nonempty Zariski open subset in $k^{*}$. We pick any $v \in k_{\text {reg }}^{*}$ and set $s=\lambda_{\tilde{e}}(v), \tilde{\mathcal{Y}}=\mathcal{Y}\left(\lambda_{\tilde{e}}, \tilde{e}\right), L=Z_{G}\left(\lambda_{\tilde{e}}\right)$. By [29, Sect. 2], the orbit $(\operatorname{Ad} L) \tilde{e}$ is open in $\mathfrak{g}\left(2 ; \lambda_{\tilde{e}}\right)$. Since $\tilde{\mathcal{Y}}$ is $L$-stable and Zariski closed, we then have $\left(s, \mathfrak{g}\left(2 ; \lambda_{\tilde{e}}\right)\right) \subset \tilde{\mathcal{Y}}$. Since $e \in \mathfrak{g}\left(2 ; \lambda_{\tilde{e}}\right)$ by our discussion in (3.4), we deduce that $(s, e) \in \tilde{\mathcal{Y}}$.

Let $U(s)$ denote the centraliser of $s$ in $R_{u}\left(Z_{G}(e)\right)$. As $s$ is semisimple and $R_{u}\left(Z_{G}(e)\right)$ is a connected unipotent group, $U(s)$ is connected too; see [4, Chap. III, (9.3)]. Since Lie $U(s)=\left(\text { Lie } R_{u}\left(Z_{G}(e)\right)\right)^{s}$, by [4,Chap. III, (9.4)], and since $b_{i}(\operatorname{Ad} s) \neq 1$ for all $i$, it must be that $U(s)=\{1\}$. As $s$ normalises the unipotent group $R_{u}\left(Z_{G}(e)\right)$, the orbit (Int $\left.R_{u}\left(Z_{G}(e)\right)\right) s$ is Zariski closed in $s R_{u}\left(Z_{G}(e)\right)$. By the preceding remark, it has the same dimension as $s R_{u}\left(Z_{G}(e)\right)$. The irreducibility of $s R_{u}\left(Z_{G}(e)\right)$ now yields $\left(s R_{u}\left(Z_{G}(e)\right), e\right) \subset \tilde{\mathcal{Y}}$. Since this holds for all $v \in k_{\text {reg, }}^{*}$ we get $\left(\lambda_{\tilde{e}}\left(k_{\text {reg }}^{*}\right) y, e\right) \subset \tilde{\mathcal{Y}}$ for all $y \in R_{u}\left(Z_{G}(e)\right)$. But then $\left(\lambda_{\tilde{e}}\left(k^{*}\right) y, e\right)=\overline{\left(\lambda_{\tilde{e}}\left(k_{\text {reg }}^{*}\right) y, e\right)} \subset \tilde{\mathcal{Y}}$ for all $y \in R_{u}\left(Z_{G}(e)\right)$, and the claim follows.

(3) Proposition 3.6 now shows that $\left(R_{u}\left(Z_{G}(e)\right), e\right) \subset \tilde{\mathcal{Y}} \cap \mathfrak{X}=\mathcal{Y}_{0}\left(\lambda_{\tilde{e}}, \tilde{e}\right)$. Combined with Lemma 3.5 this implies that $\left(\right.$ Lie $\left.R_{u}\left(Z_{G}(e)\right), e\right) \subset \tilde{\eta}\left(\mathcal{Y}_{0}\left(\lambda_{\tilde{e}}, \tilde{e}\right)\right) \subseteq \mathfrak{C}^{\text {nil }}(\mathfrak{g})$ and all irreducible components of the Zariski closed set $\tilde{\eta}\left(\mathcal{Y}_{0}\left(\lambda_{\tilde{e}}, \tilde{e}\right)\right)$ have dimension $n$.

Let $Z$ be an irreducible component of $\tilde{\eta}\left(\mathcal{Y}_{0}\left(\lambda_{\tilde{e}}, \tilde{e}\right)\right)$ containing (Lie $\left.R_{u}\left(Z_{G}(e)\right), e\right)$. Then $Z \subseteq \mathfrak{C}\left(e^{\prime}\right)$ for some $e^{\prime} \in \mathcal{N}$; see Proposition 3.3. As $\operatorname{dim} Z=n$ it must be that $\operatorname{dim} \mathfrak{C}\left(e^{\prime}\right) \geq n$. Our discussion in (3.1) now shows that $e^{\prime}$ is distinguished in $\mathfrak{g}$ and $Z=\mathfrak{C}\left(e^{\prime}\right)$. Since $e$ is almost distinguished, we also have $\mathcal{N}(\mathfrak{z}(e ; 0))=\{0\}$ and $\mathcal{N} \cap \mathfrak{z}(e)=\bigoplus_{i>0} \mathfrak{z}(e ; i)=$ Lie $R_{u}\left(Z_{G}(e)\right)$; see (3.1). As $\mathfrak{C}\left(e^{\prime}\right)$ is $G$-stable, we then have $G \cdot(\mathcal{N} \cap \mathfrak{z}(e), e) \subseteq \mathfrak{C}\left(e^{\prime}\right)$. Since $\mathfrak{C}\left(e^{\prime}\right)$ is a component of $\mathfrak{C}^{\text {nil }}(\mathfrak{g})$, it is stable under the involution $(x, y) \mapsto(y, x)$ on $\mathfrak{g} \times \mathfrak{g}$. But then $\mathfrak{C}(e) \subseteq \mathfrak{C}\left(e^{\prime}\right)$ completing the proof. 


\section{Applications and concluding remarks}

4.1. Let $S$ be a smooth projective surface over $k$ and let $\operatorname{Hilb}^{n}(S)$ be the Hilbert scheme parametrising the 0-dimensional subschemes of $S$ of length $n$. The scheme $\operatorname{Hilb}^{n}(S)$ is known to be smooth and projective; see [8, 9]. Let $\operatorname{Sym}^{n}(S)$ denote the $n$ fold symmetric power of $S$, the geometric quotient of $S^{n}$ by the permutation action of $\mathfrak{S}_{n}$. By assigning to any 0-dimensional subscheme of $S$ its support with multiplicities one obtains a map

$$
w_{n}: \operatorname{Hilb}^{n}(S) \longrightarrow \operatorname{Sym}^{n}(S)
$$

called the Hilbert-Chow morphism. According to [8, 9], this map provides a natural desingularisation of $\operatorname{Sym}^{n}(S)$. If $z$ is a point of $\operatorname{Sym}^{n}(S)$ representing the effective 0 -cycle $\sum n_{i}\left[s_{i}\right]$ with $\sum n_{i}=n$, then the fibre $w_{n}^{-1}(z)$ is isomorphic to $\prod_{i} \operatorname{Hilb}^{n_{i}} \mathcal{O}_{S, s_{i}}$ where $\mathrm{Hilb}^{r} \mathcal{O}_{S, s}$ is the punctual Hilbert scheme parametrising the ideals of colength $r$ in the local ring $\mathcal{O}_{S, s}$.

For any $s \in S$ the scheme $\left(\operatorname{Hilb}^{r} \mathcal{O}_{S, s}\right)_{\text {red }}$ is isomorphic to the (reduced) Hilbert scheme $\mathcal{H}_{r}=\operatorname{Hilb}^{r} k[[x, y]]$ parametrising the ideals of colength $d$ in the ring of formal power series $k[[x, y]]$; see [14, Chap. 1]. This shows that the punctual Hilbert schemes $\mathcal{H}_{r}$ with $r \leq n$ serve as the building blocks for all fibres of the Hilbert-Chow morphism.

Let $\mathfrak{m}$ be the maximal ideal of $k[[x, y]]$ and $A=k[[x, y]] / \mathfrak{m}^{r}$, a finite dimensional local algebra over $k$. Let $\operatorname{Gr}^{r}(A)$ denote the Grassmannian of all $k$-subspaces of codimension $r$ in $A$. By [14, Chap. 1], any ideal $I$ of colength $r$ in $k[[x, y]]$ contains $\mathfrak{m}^{r}$ and the map $I \mapsto I / \mathfrak{m}^{r}$ identifies $\mathcal{H}_{r}$ with a closed subset of $\operatorname{Gr}^{r}(A)$. The algebraic variety structure on $\mathcal{H}_{r}$ is given by this identification.

Now let $V$ is an $r$-dimensional vector space over $k$ and $\mathfrak{g}=\mathfrak{g l}(V)$. Observe that $G=\mathrm{GL}(V)$ acts on $\mathfrak{C}^{\text {nil }}(\mathfrak{g}) \times V$ by $g \cdot(a, b ; v)=\left(g a g^{-1}, g b g^{-1} ; g(v)\right)$ for all $g \in G$ and all $(a, b ; v) \in \mathfrak{C}^{\text {nil }}(\mathfrak{g}) \times V$. Let $U$ be the Zariski open subset in $\mathfrak{C}^{\text {nil }}(\mathfrak{g}) \times V$ consisting of all $(a, b ; v)$ such that $a^{i} b^{j}(v)$ with $i, j \in \mathbb{Z}_{+}$span $V$. Clearly, $U$ is $G$-stable. As observed in [22, 2], the morphism $\psi: U \longrightarrow \operatorname{Gr}^{r}(A)$ assigning to $(a, b ; v) \in U$ the point $\{\phi \in A \mid \phi(a, b)(v)=0\}$ on $\operatorname{Gr}^{r}(A)$, maps $U$ onto $\mathcal{H}_{r}$. Moreover, $\operatorname{GL}(V)$ acts freely on $U$ and the fibres of $\psi$ are exactly the $\operatorname{GL}(V)$-orbits on $U$.

It is immediate from Theorems 2.5 and 3.7 that $\mathfrak{C}^{\text {nil }}(\mathfrak{g}) \times V$ is an irreducible variety of dimension $r^{2}+r-1$. Then so is $U$ being open dense in $\mathfrak{C}^{\text {nil }}(\mathfrak{g}) \times V$. Combining the theorem on the dimension of the fibres of a morphism with the discussion above we now obtain the following:

Corollary 4.1. If $k$ is an algebraically closed field of characteristic $p \geq 0$, then the punctual Hilbert scheme $\mathcal{H}_{r}$ is irreducible over $k$ and $\operatorname{dim} \mathcal{H}_{r}=r-1$.

This corollary generalises earlier results by Briançon [6] for $p=0$, Iarrobino [14] for $p>n$, and Basili [1] for $p \geq n / 2$. In conjunction with an observation in [22, (1.2)] it also shows that $\mathcal{H}_{r}$ is isomorphic (as a reduced scheme) to the closure in $\operatorname{Gr}^{r}(A)$ of the $(r-1)$-dimensional family of principal ideals

$$
\mathcal{J}=\left\{A\left(x-\left(t_{1} y+t_{2} y^{2}+\cdots+t_{r-1} y^{r-1}\right)\right) \mid\left(t_{1}, t_{2}, \ldots, t_{r-1}\right) \in \mathbb{A}^{r-1}\right\} .
$$

4.2. In this subsection we assume that $k$ is an algebraically closed field of characteristic $p>0$. Let $\mathcal{L}$ be a finite dimensional restricted Lie algebra over $k$ with $p$ th power map $x \mapsto x^{[p]}$. We denote by $\mathcal{N}(\mathcal{L})$ the nilpotent variety of $\mathcal{L}$, the set of all $x \in \mathcal{L}$ 
with $x^{[p]^{N}}=0$ for $N \gg 0$. It is immediate from Jacobson's formula [15, Chap. V, Sect. 7] that the map $\pi: \mathcal{L} \rightarrow \mathcal{L}, x \mapsto x^{[p]}$, is a morphism given by homogeneous polynomial functions on $\mathcal{L}$ of degree $p$.

Recall that an element $x \in \mathcal{L}$ is semisimple if it lies in the restricted subalgebra of $\mathcal{L}$ generated by $x^{[p]}$. Let $\mathcal{L}_{s s}$ denote the set of all semisimple elements of $\mathcal{L}$. Using the Jordan-Chevalley decomposition in $\mathcal{L}$ it is easy to observe that $\mathcal{L}_{s s}$ coincides with $\pi^{N}(\mathcal{L})$ for $N \gg 0$. We define

$$
e(\mathcal{L}):=\min \left\{r \in \mathbb{Z}_{+} \mid \pi^{r}(W) \subseteq \mathcal{L}_{s s} \text { for some open dense } W \subseteq \mathcal{L}\right\} .
$$

Recall that a restricted subalgebra $\mathfrak{t} \subseteq \mathcal{L}$ is called toral (or a torus) if the $p$ th power map of $\mathcal{L}$ is injective on $\mathfrak{t}$. By [15, Chap. V, Sect. 8], any torus $\mathfrak{t}$ in $\mathcal{L}$ is abelian and spanned by its subset $\mathfrak{t}^{\text {tor }}:=\left\{t \in \mathfrak{t} \mid t^{[p]}=t\right\}$. We define

$$
M T(\mathcal{L}):=\max \{\operatorname{dim} \mathfrak{t} \mid \mathfrak{t} \text { is toral in } \mathcal{L}\} .
$$

Note that $M T(\mathcal{L})=0$ if and only if $\mathcal{L}=\mathcal{N}(\mathcal{L})$, while from the main results of [25] it follows that $e(\mathcal{L})=0$ if and only if $\mathcal{L}$ possesses a toral Cartan subalgebra (see also [26, Theorem 1]). Let $n=\operatorname{dim} \mathcal{L}, e=e(\mathcal{L}), s=M T(\mathcal{L})$, and let $v_{1}, \ldots, v_{n}$ be a basis of $\mathcal{L}$. According to [26, Theorem 2], there exist homogeneous polynomials $\psi_{0}, \ldots, \psi_{s-1} \in k\left[X_{1}, \ldots, X_{n}\right]$ with $\operatorname{deg} \psi_{i}=p^{s+e}-p^{i+e}$ and the property that for any $x=\sum_{i=1}^{n} x_{i} v_{i}$ one has

$$
x^{[p]^{s+e}}=\sum_{i=0}^{s-1} \psi_{i}(x) x^{[p]^{i+e}} .
$$

We view $\psi_{0}, \ldots, \psi_{s-1}$ as polynomial functions on $\mathcal{L}$.

Theorem 4.2. The following are true:

1. $\psi_{i}\left(x^{[p]}\right)=\psi_{i}(x)^{p}$ for all $x \in \mathcal{L}$ and all $i \leq s-1$.

2. $\mathcal{N}(\mathcal{L})$ coincides with the set of all common zeros of $\psi_{0}, \ldots, \psi_{s-1}$ in $\mathcal{L}$.

3. All irreducible components of $\mathcal{N}(\mathcal{L})$ have dimension $n-s$.

Proof. (1) Let $g_{i+1}=\psi_{i}^{p}-\psi_{i} \circ \pi$ where $0 \leq i \leq s-1$. Then $g_{i+1}$ is a polynomial function on $\mathcal{L}$. Applying $\pi$ to both sides of $(7)$ we get $x^{[p]^{s+e+1}}=\sum_{i=0}^{s-1} \psi_{i}(x)^{p} x^{[p]^{i+e+1}}$ while substituting in (7) $x$ by $x^{[p]}$ we get $x^{[p]^{s+e+1}}=\sum_{i=0}^{s-1} \psi_{i}\left(x^{[p]}\right) x^{[p]^{i+e+1}}$. Hence

$$
\sum_{i=1}^{s} g_{i}(x) x^{[p]^{e+i}}=0 \quad(\forall x \in \mathcal{L}) .
$$

For $r \in \mathbb{N}$ and $x \in \mathcal{L}$ write $\pi^{r}(x)=\sum_{i=1}^{n} \pi_{r, i}(x) v_{i}$ where $\pi_{r, i}$ is a homogeneous polynomial function on $\mathcal{L}$ of degree $p^{r}$. As explained in $[26, \S 1]$ the matrix

$$
\left(\begin{array}{cccc}
\pi_{e, 1} & \pi_{e, 2} & \cdots & \pi_{e, n} \\
\pi_{e+1,1} & \pi_{e+1,2} & \cdots & \pi_{e+1, n} \\
\vdots & \vdots & & \vdots \\
\pi_{e+s-1,1} & \pi_{e+s-1,2} & \cdots & \pi_{e+s-1, n}
\end{array}\right)
$$

has rank $s$. Hence there is an open dense subset $U \subset \mathcal{L}$ with the property that $u^{[p]^{e}}, u^{[p]^{e+1}}, \ldots, u^{[p]^{e+s-1}}$ are linearly independent for all $u \in U$. Let $W$ be an open dense set in $\mathcal{L}$ with $\pi^{e}(W) \subset \mathcal{L}_{s s}$. Note that for each $u \in W$ the linear span of $u^{[p]^{j}}$ with $j \geq e$ is a torus. It follows that $u^{\left[p^{e+1}\right.}, u^{[p]^{e+2}}, \ldots, u^{[p]^{e+s}}$ are linearly independent whenever $u \in U \cap W$. So (8) shows that all $g_{i}$ vanish on $U \cap W$. As $U \cap W$ is open dense in $\mathcal{L}$, we get $\psi_{i} \circ \pi=\psi_{i}^{p}$ for all $i \leq s-1$. 
(2) Let $\mathcal{Z}$ denote the set of all common zeros of $\psi_{0}, \ldots, \psi_{s-1}$ in $\mathcal{L}$. It is immediate from $(7)$ that $\mathcal{N}(\mathcal{L}) \supseteq \mathcal{Z}$. On the other hand, if $x \in \mathcal{N}(\mathcal{L})$, then

$$
\psi_{i}(x)^{p^{N}}=\psi_{i}\left(x^{[p]^{N}}\right)=\psi_{i}(0)=0 \quad(0 \leq i \leq s-1)
$$

provided $N \gg 0$. Hence $\mathcal{Z}=\mathcal{N}(\mathcal{L})$ implying that each irreducible component of $\mathcal{N}(\mathcal{L})$ has dimension $\geq n-s$, by the affine dimension theorem. If $\mathcal{N}(\mathcal{L})$ had a component of dimension $>n-s$, then it would intersect nontrivially with any $s$ dimensional torus in $\mathcal{L}$ (again by the affine dimension theorem). But this is impossible as no nonzero element in $\mathcal{L}$ can be both nilpotent and semisimple. Thus all irreducible components of $\mathcal{N}(\mathcal{L})$ are of dimension $n-s$.

Remark. It is conjectured in [26] that the variety $\mathcal{N}(\mathcal{L})$ is always irreducible. This conjecture is still open; it is not clear at present how to approach it without using the structure theory of finite dimensional restricted Lie algebras.

4.3. We are going to apply Theorem 4.2 for estimating the dimension of $\mathfrak{C}^{\text {nil }}(\mathfrak{g})$ in a more general setting. We assume in this subsection that $k$ is an algebraically closed field of characteristic $p>0$ and $G$ is a connected reductive $k$-group (so we allow $p$ to be a bad prime from now on). We adopt the notation introduced in Sections 2 and 3.

Let $e_{1}, \ldots, e_{m}$ be representatives of the nilpotent $G$-orbits in $\mathfrak{g}$; these orbits are finite in number, by [13]. Then $\mathfrak{C}^{\text {nil }}(\mathfrak{g})=\mathfrak{C}\left(e_{1}\right) \cup \ldots \cup \mathfrak{C}\left(e_{m}\right)$, as before, but in the present case there is no guaranty that the $\mathfrak{C}\left(e_{i}\right)$ 's are all irreducible. However, the nilpotent variety $\mathcal{N}$ of $\mathfrak{g}$ is still irreducible, by [4, Chap. IV, (14.16)]. Combined with [13] this implies that $\mathcal{N}$ contains a unique open $G$-orbit. Let $e_{\text {reg }}$ be a representative of this orbit.

Proposition 4.3. The following are true:

1. For any $e \in \mathcal{N}$ the variety $\mathfrak{C}(e)$ is equidimensional and

$$
\operatorname{dim} \mathfrak{C}(e)=\operatorname{dim} G-\operatorname{dim} Z_{G}(e)+\operatorname{dim} \mathfrak{z}(e)-M T(\mathfrak{z}(e)) .
$$

2. $\mathfrak{C}\left(e_{\text {reg }}\right)$ is an irreducible component of $\mathfrak{C}^{\text {nil }}(\mathfrak{g})$ and

$$
\operatorname{dim} \mathfrak{C}\left(e_{\text {reg }}\right)=\operatorname{dim} G-\operatorname{rk} G+\operatorname{dim} \mathfrak{z}\left(e_{\text {reg }}\right)-\operatorname{dim} \mathfrak{z}(\mathfrak{g}) .
$$

Proof. (1) Let $M_{1}, \ldots, M_{s}$ be the irreducible components of $\mathcal{N}(\mathfrak{z}(e))$. By Theorem $4.2(3), \operatorname{dim} M_{i}=\operatorname{dim} \mathfrak{z}(e)-M T(\mathfrak{z}(e))$ for all $i \leq s$. Let $\tilde{M}_{i}$ denote the Zariski closure of $G \cdot\left(e, M_{i}\right)$ in $\mathfrak{g} \times \mathfrak{g}$. Then $\mathfrak{C}(e)=\tilde{M}_{1} \cup \ldots \cup \tilde{M}_{s}$. It follows that each irreducible component of $\mathfrak{C}(e)$ has the form $\tilde{M}_{j}$ for some $j \leq s$. The map

$$
\left.\xi: G \times M_{j} \longrightarrow \tilde{M}_{j}, \quad(x, m) \longmapsto((\operatorname{Ad} x) e,(\operatorname{Ad} x) m)\right),
$$

is a dominant morphism of algebraic varieties. The fibre $\xi^{-1}(\xi(g, a))$ consists of all $(x, m) \in G \times M_{j}$ with $g^{-1} x \in Z_{G}(e)$ and $\left(\operatorname{Ad} x^{-1} g\right) a=m$. From this it is immediate that $\xi^{-1}(\xi(g, a)) \cong \operatorname{Tran}\left(a, M_{j}\right):=\left\{z \in Z_{G}(e) \mid(\operatorname{Ad} z) a \in M_{j}\right\}$. Since $Z_{G}(e)$ permutes the components $M_{1}, \ldots, M_{s}$, the connected component $Z_{G}(e)^{\circ}$ acts on Tran $\left(a, M_{j}\right)$ by left translations. Since $Z_{G}(e)^{\circ}$ has finite index in $Z_{G}(e)$, it follows that $\operatorname{Tran}\left(a, M_{j}\right)$ and $Z_{G}(e)$ have the same dimension. As a result, $\operatorname{dim} \xi^{-1}(\xi(g, a))=$ $\operatorname{dim} Z_{G}(e)$. The theorem on the dimension of the fibres of a morphism now gives

$$
\begin{aligned}
\operatorname{dim} \tilde{M}_{j} & =\operatorname{dim} G+\operatorname{dim} M_{j}-\operatorname{dim} Z_{G}(e) \\
& =\operatorname{dim} G-\operatorname{dim} Z_{G}(e)+\operatorname{dim} \mathfrak{z}(e)-M T(\mathfrak{z}(e)) \\
& 17
\end{aligned}
$$


proving the first part of the proposition. As $\operatorname{dim} Z_{G}\left(e_{\mathrm{reg}}\right)=\operatorname{rk} G$, we also obtain

$$
\operatorname{dim} \mathfrak{C}\left(e_{\text {reg }}\right)=\operatorname{dim} G-\operatorname{rk} G+\operatorname{dim} \mathfrak{z}\left(e_{\text {reg }}\right)-M T\left(\mathfrak{z}\left(e_{\text {reg }}\right)\right) .
$$

(2) Let $T$ be a maximal torus in $G$ and $\mathfrak{t}=$ Lie $T$. Let $\left\{\alpha_{1}, \ldots, \alpha_{l}\right\}$ be a basis of simple roots in the root system of $G$ relative to $T$. According to [16, Prop. 4.14], it can be assumed that $e_{\text {reg }}=e_{\alpha_{1}}+\ldots+e_{\alpha_{l}}$ where $e_{\alpha_{i}}$ are simple root vectors in $\mathfrak{g}$ (the argument in [16] is based on [7, Prop. 5.8.5]) which is applicable in any characteristic). There is a cocharacter $\lambda_{\text {reg }} \in X_{*}(T)$ such that $\alpha_{i}\left(\lambda_{\text {reg }}(t)\right)=t^{2}$ for all $i$ and all $t \in k^{*}$. It is easy to see that $\mathfrak{g}\left(0 ; \lambda_{\text {reg }}\right)=\mathfrak{t}$. For $r \in \mathbb{Z}$, we set $\mathfrak{z}\left(e_{\text {reg }} ; r\right)=\mathfrak{z}\left(e_{\text {reg }}\right) \cap \mathfrak{g}\left(r ; \lambda_{\text {reg }}\right)$. Then $\mathfrak{z}\left(e_{\text {reg }} ; 0\right)$ consists of all $x \in \mathfrak{t}$ with $\left(\mathrm{d} \alpha_{i}\right)_{e}(x)=0$ for all $i$. Since any root $\gamma$ is a $\mathbb{Z}$-linear combination of simple roots, the root function $(\mathrm{d} \gamma)_{e} \in \mathfrak{t}^{*}$ is an $\mathbb{F}_{p}$-linear combination of the root functions $\left(\mathrm{d} \alpha_{i}\right)_{e}$. It follows that any root function $(\mathrm{d} \gamma)_{e}$ vanishes on $\mathfrak{z}\left(e_{\text {reg }} ; 0\right) \subseteq \mathfrak{t}$. Since $\mathfrak{t}$ is abelian, this implies that $\mathfrak{z}\left(e_{\text {reg }} ; 0\right) \subseteq \mathfrak{z}(\mathfrak{g})$. On the other hand, decomposing $\mathfrak{z}(\mathfrak{g})$ into weight spaces with respect to $T$ one observes without difficulty that $\mathfrak{z}(\mathfrak{g}) \subseteq \mathfrak{t}$. This yields $\mathfrak{z}\left(e_{\text {reg }} ; 0\right)=\mathfrak{z}(\mathfrak{g})$.

As a result, for any $r \in \mathbb{Z}$ the set $\mathfrak{Z}_{r}:=\left\{\operatorname{ad} x \mid x \in \mathfrak{z}\left(e_{\text {reg }} ; r\right)\right\}$ consists of nilpotent endomorphisms of $\mathfrak{g}$. Since $\left[\mathfrak{Z}_{i}, \mathfrak{Z}_{j}\right] \subseteq \mathfrak{Z}_{i+j}$ for all $i, j \in \mathbb{Z}$, the union $\bigcup_{r \in \mathbb{Z}} \mathfrak{Z}_{r}$ is a weakly closed nilset in End $\mathfrak{g}$. Applying Jacobson's theorem on weakly closed nilsets, see [15, Chap. II, $\S 2$, Theorem 1], we now obtain that the associative subalgebra generated by the $\mathfrak{Z}_{i}$ 's is nilpotent. But then $\mathfrak{z}\left(e_{\text {reg }}\right)$ is a (restricted) nilpotent subalgebra of $\mathfrak{g}$, by Engel's theorem. It follows that the set $\mathfrak{z}\left(e_{\text {reg }}\right)_{s s}$ is central and, moreover, a unique maximal torus in $\mathfrak{z}\left(e_{\text {reg }}\right)$. Since $\mathfrak{z}\left(e_{\text {reg }}\right)_{s s}$ is stable under $\operatorname{Ad} \lambda_{\text {reg }}\left(k^{*}\right)$, it must be that $\mathfrak{z}\left(e_{\text {reg }}\right)_{s s}=\mathfrak{z}(\mathfrak{g})$. As a consequence,

$$
\operatorname{MT}\left(\mathfrak{z}\left(e_{\text {reg }}\right)\right)=\operatorname{dim} \mathfrak{z}(\mathfrak{g}) .
$$

(3) Thanks to (9) and (10) we are now reduced to show that $\mathfrak{C}\left(e_{\text {reg }}\right)$ is a component of $\mathfrak{C}^{\text {nil }}(\mathfrak{g})$. Let us first show that $\mathfrak{C}\left(e_{\text {reg }}\right)$ is irreducible. Let $\overline{\mathfrak{z}}=\mathfrak{z}\left(e_{\text {reg }}\right) / \mathfrak{z}(\mathfrak{g})$ and let $\beta: \mathfrak{z}\left(e_{\text {reg }}\right) \rightarrow \overline{\mathfrak{z}}$ denote the canonical homomorphism.Using the Jordan-Chevalley decomposition in $\mathfrak{z}\left(e_{\text {reg }}\right)$ and our final remarks in part 2 one easily observes that $\beta$ induces a bijective morphism $\mathcal{N}\left(\mathfrak{z}\left(e_{\text {reg }}\right)\right) \rightarrow \overline{\mathfrak{z}}$. The latter, in turn, induces a bijective morphism $\mathbb{P}\left(\mathcal{N}\left(\mathfrak{z}\left(e_{\text {reg }}\right)\right)\right) \longrightarrow \mathbb{P}(\overline{\mathfrak{z}})$ of projective varieties. Since $\mathbb{P}(\overline{\mathfrak{z}})$ is obviously irreducible, so must be $\mathbb{P}\left(\mathcal{N}\left(\mathfrak{z}\left(e_{\text {reg }}\right)\right)\right)$, by [32, Chap. I, $\S 6$, Theorem 8]. But then $\mathcal{N}\left(\mathfrak{z}\left(e_{\text {reg }}\right)\right)$ is irreducible as well, and hence so is $\mathfrak{C}\left(e_{\text {reg }}\right)$.

Let $Z$ be an irreducible component of $\mathfrak{C}^{\text {nil }}(\mathfrak{g})$ containing $\mathfrak{C}\left(e_{\text {reg }}\right)$. By part $1, Z=$ $\overline{G \cdot\left(e, M_{j}\right)}$ where $e \in \mathcal{N}$ and $M_{j}$ is a component on $\mathcal{N}(\mathfrak{z}(e))$. It follows that the first projection $\mathrm{pr}_{1}: \mathfrak{g} \times \mathfrak{g} \rightarrow \mathfrak{g}, \quad(x, y) \mapsto x$ takes $Z$ to the Zariski closure of the $G$-orbit of $e$. On the other hand, $\operatorname{pr}_{1}\left(\mathfrak{C}\left(e_{\text {reg }}\right)\right)$ contains the $G$-orbit of $e_{\text {reg }}$, an open subset in $\mathcal{N}$. This shows that $e$ is $G$-conjugate to $e_{\text {reg. }}$. Therefore, $M_{j}=\mathcal{N}\left(\mathfrak{z}\left(e_{\text {reg }}\right)\right)$ and $Z=\mathfrak{C}\left(e_{\text {reg }}\right)$, completing the proof.

Remark. It is quite possible that $\mathfrak{C}^{\text {nil }}(\mathfrak{g})$ is equidimensional for any connected reductive group $G$ and for any $p$. If this is the case, then $\operatorname{dim} \mathfrak{C}^{\text {nil }}(\mathfrak{g})=\operatorname{dim} \mathfrak{C}\left(e_{\text {reg }}\right)$, by Proposition 4.3. In [34, Sect. 2], Springer essentially computed $\operatorname{dim} \mathfrak{z}\left(e_{\text {reg }}\right)$ for any semisimple group $G$ in bad characteristic. In view of Proposition 4.3(2), this computation yields a close formula for $\operatorname{dim} \mathfrak{C}\left(e_{\text {reg }}\right)$.

Proposition 4.3 also shows that if $\mathfrak{C}^{\text {nil }}(\mathfrak{g})$ is equidimensional, then

$$
\operatorname{dim} \mathfrak{z}(e)-\operatorname{dim} Z_{G}(e)-M T(\mathfrak{z}(e)) \leq \operatorname{dim} \mathfrak{z}\left(e_{\text {reg }}\right)-\operatorname{rk} G-\operatorname{dim} \mathfrak{z}(\mathfrak{g})
$$


for any $e \in \mathcal{N}$. Thus proving the equidimensionality of $\mathfrak{C}^{\text {nil }}(\mathfrak{g})$ in bad characteristic would have important implications for the detailed study of the adjoint action of $G$.

\section{Appendix}

Here we carry out the case-by-case analysis involved in the proof of Proposition 3.2 (we are assuming for a contradiction that $e$ is not almost distinguished in $\mathfrak{g}$ ).

(2) Suppose that $G$ is a group of type B, C or D. Since $p$ is good for $G$ we have $p \neq 2$. Assume that $\operatorname{dim} V \geq 2$ and let $\Psi$ be a nondegenerate bilinear form on $V$ such that $\Psi(u, v)=(-1)^{\varkappa} \Psi(v, u)$ for all $u, v \in V$, where $\varkappa \in\{0,1\}$. Let $G(\Psi)$ be the closed subgroup of $\mathrm{SL}(V)$ consisting of all $g \in \mathrm{SL}(V)$ with $\Psi(g(u), g(v))=\Psi(u, v)$ for all $u, v \in V$. Then

$$
\mathfrak{g}(\Psi):=\{x \in \mathfrak{s l}(n) \mid \Psi(x(u), v)+\Psi(u, x(v))=0 \text { for all } u, v \in V\}
$$

is the Lie algebra of $G(\Psi)$. It is well-known that $G$ is isogenic to $G(\Psi)$ for a suitable choice of $V$ and $\Psi$, and $\mathfrak{g} \cong \mathfrak{g}(\Psi)$ as restricted Lie algebras. We thus may identify $\mathfrak{g}$ with $\mathfrak{g}(\Psi)$ and view our $e \in \mathcal{N}$ as a nilpotent endomorphism of $V$.

Let $\left(n_{1} \geq n_{2} \geq \cdots \geq n_{s}\right)$ be the partition of $\operatorname{dim} V$ associated with $e$. According to $\left[35\right.$, Chap. IV , (2.19)] there is a direct sum decomposition $V=V_{1} \oplus \cdots \oplus V_{s}$ with $\operatorname{dim} V_{i}=n_{i}$ for all $i$, such that

1. each $V_{i}$ is $e$-stable and $e$ acts on $V_{i}$ as a nilpotent Jordan block of order $n_{i}$;

2. if $n_{i}+\varkappa$ is odd, then $\Psi$ is nondegenerate on $V_{i}$ and $\Psi\left(V_{i}, V_{k}\right)=0$ for $k \neq i$;

3. if $n_{i}+\varkappa$ is even, then $\Psi$ vanishes on $V_{i} \times V_{i}$ and there exists a unique $i^{*}=i \pm 1$ such that $n_{i^{*}}=n_{i}, \Psi$ is nondegenerate on $V_{i} \oplus V_{i^{*}}$, and $V_{k}$ is orthogonal to $V_{i} \oplus V_{i^{*}}$ for $k \notin\left\{i, i^{*}\right\}$.

For $i \leq \operatorname{dim} V$ we denote by $r(i)$ the number of $k$ with $n_{k}=i$. Let $I_{1}$ (respectively, $I_{2}$ ) be the set of all $i \in\{1, \ldots, s\}$ such that $n_{i}+\varkappa$ is odd (respectively, even) and $r\left(n_{i}\right) \geq 3$ (respectively, $r\left(n_{i}\right) \geq 2$ ). Put $I=I_{1} \cup I_{2}$. Our assumption on $e$ implies that $I \neq \emptyset$; see [35, Chap. IV, (2.25)]. Let $j$ be the smallest integer in $I$ and set $d:=n_{j}=\operatorname{dim} V_{j}$.

(a) First suppose that $d+\varkappa$ is odd. Then $r(d) \geq 3 \operatorname{implying} \operatorname{dim} V_{j}=\operatorname{dim} V_{j+1}=$ $\operatorname{dim} V_{j+2}=d$. As $d+\varkappa$ is odd, the subspaces $V_{j}, V_{j+1}$ and $V_{j+2}$ are orthogonal to each other relative to $\Psi$. Let $M=V_{j} \oplus V_{j+1} \oplus V_{j+2}$. For $t \in\{0,1,2\}$, there is a basis $m_{1, t}, \ldots, m_{d, t}$ of $V_{j+t}$ such that $e\left(m_{d, t}\right)=0, e\left(m_{i, t}\right)=m_{i+1, t}$ for $1 \leq i \leq d-1$, and

$$
\Psi\left(m_{i, t}, m_{k, t}\right)=(-1)^{i-1} \delta_{i, d+1-k} \quad(1 \leq i, k \leq d) ;
$$

see [35, Chap. IV, (2.19)]. For $1 \leq i \leq d$, define $u_{i}:=\frac{1}{\sqrt{2}}\left(m_{i, 0}+\sqrt{-1} m_{i, 2}\right), v_{i}:=m_{i, 1}$, $w_{i}:=-\frac{1}{\sqrt{2}}\left(m_{i, 0}-\sqrt{-1} m_{i, 2}\right)$ and let $u_{i}=v_{i}=w_{i}=0$ for all $i>d$. Let $U$ and $W$ be the linear spans of the $u_{i}$ 's and $w_{i}$ 's, respectively. By construction, these subspaces are totally isotropic with respect to $\Psi$. Furthermore, $M=U \oplus V_{j+1} \oplus W$ and $\Psi$ vanishes on $(U+W) \times V_{j+1}$. Finally, $\Psi\left(u_{i}, w_{k}\right)=-(-1)^{i-1} \delta_{i, d+1-k}=-\Psi\left(v_{i}, v_{k}\right)$ for all $i, k \leq d$. There is $z \in \mathfrak{g l}(M)$ such that $z\left(u_{i}\right)=v_{i}, z\left(v_{i}\right)=w_{i}$ and $z\left(w_{i}\right)=u_{i+1}$ for all $i$. It acts on $M$ as a nilpotent Jordan block of order $3 d$. Similar to part 1 of this proof one checks that the endomorphisms $e_{\mid M}$ and $z$ commute. Next observe 
that $\Psi\left(z\left(u_{i}\right), v_{k}\right)=\Psi\left(v_{i}, v_{k}\right)=-\Psi\left(u_{i}, w_{k}\right)=-\Psi\left(u_{i}, z\left(v_{k}\right)\right)$ and

$$
\begin{aligned}
\Psi\left(z\left(w_{i}\right), w_{k}\right) & =\Psi\left(u_{i+1}, w_{k}\right)=-(-1)^{i} \delta_{i+1, d+1-k}=(-1)^{d+\varkappa-i} \delta_{i+1, d+1-k} \\
& =(-1)^{k+\varkappa} \delta_{k+1, d+1-i}=-(-1)^{\varkappa} \Psi\left(u_{k+1}, w_{i}\right)=-\Psi\left(w_{i}, z\left(w_{k}\right)\right) .
\end{aligned}
$$

Combined with our earlier remarks this shows that the endomorphism $z$ is skewadjoint with respect to $\Psi_{\mid M \times M}$. Property 2 of our direct sum decomposition now ensures that there exists $\hat{z} \in \mathfrak{g}=\mathfrak{g}(\Psi)$ such that $\hat{z}_{\mid M}=z$ and $\hat{z}_{\mid V_{i}}=e_{\mid V_{i}}$ for all $i \notin\{j, j+1, j+2\}$. By construction, $\hat{z} \in \mathcal{N} \cap \mathfrak{z}(e)$ and the partition of $\operatorname{dim} V$ associated with $\hat{z}$ is not dominated by that of $e$. So $\hat{z} \notin \overline{\operatorname{GL}(V) e}$, by [10]. But then $\hat{z} \notin \overline{G e}$, a contradiction.

(b) Now suppose $d+\varkappa$ is even. This case is harder as we do not have much room for manoeuvre here (indeed, it may happen that $r(d)=2$ ). Set $M:=V_{j} \oplus V_{j^{*}}=V_{j} \oplus V_{j+1}$.

By property 3 of our decomposition, the subspaces $V_{j}$ and $V_{j+1}$ are totally isotropic with respect to $\Psi$. According to [35, Chap. IV, (2.19)] there exist bases $v_{1}, \ldots, v_{d}$ and $v_{1}^{\prime}, \ldots, v_{d}^{\prime}$ of $V_{j}$ and $V_{j+1}$ respectively, such that $e\left(v_{d}\right)=e\left(v_{d}^{\prime}\right)=0, e\left(v_{i}\right)=v_{i+1}$, $e\left(v_{i}^{\prime}\right)=v_{i+1}^{\prime}$ for $1 \leq i<d$, and

$$
\Psi\left(v_{i}, v_{k}^{\prime}\right)=(-1)^{i-1} \delta_{i, d+1-k} \quad(1 \leq i, k \leq d) .
$$

Since $d+\varkappa$ is even we have $\Psi\left(v_{i}^{\prime}, v_{k}\right)=-\Psi\left(v_{i}, v_{k}^{\prime}\right)$. For $i>d$, put $v_{i}=v_{i}^{\prime}=0$.

Let $\mathcal{A}$ be the algebra $k[X] /\left(X^{d}\right)$ and $x$ be the image of $X$ in $\mathcal{A}$. Denote by $C$ the centraliser of $e_{\mid M}$ in $\operatorname{End}(M)$. Since $e_{\mid M}$ acts on $M$ as a direct sum of two Jordan blocks of order $d$, there exists an isomorphism of associative algebras $\varphi: C \stackrel{\sim}{\longrightarrow} \operatorname{Mat}_{2}(\mathcal{A})$ such that $\varphi\left(e_{\mid M}\right)=\operatorname{diag}(x, x)$. Specifically, if $\phi_{h k}(x)$ is the $(h, k)$ th entry of $\varphi(c)$ for $c \in C$, then $c\left(v_{1}\right)=\phi_{11}(e) v_{1}+\phi_{21}(e) v_{1}^{\prime}$ and $c\left(v_{1}^{\prime}\right)=\phi_{12}(e) v_{1}+\phi_{22}(e) v_{1}^{\prime}$.

Now let $z \in C$ be such that $\varphi(z)=\left(\begin{array}{cc}x & 1 \\ x^{2} & x\end{array}\right)$. We claim that $z$ is skew-adjoint with respect to $\Psi_{\mid M \times M}$. To prove this we first observe that $z\left(v_{i}\right)=v_{i+1}+v_{i+2}^{\prime}$ and $z\left(v_{i}^{\prime}\right)=v_{i+1}^{\prime}+v_{i}$ for all $i$. Then

$$
\begin{aligned}
& \Psi\left(z\left(v_{i}^{\prime}\right), v_{k}^{\prime}\right)=\Psi\left(v_{i}, v_{k}^{\prime}\right)=-\Psi\left(v_{i}^{\prime}, v_{k}\right)=-\Psi\left(v_{i}^{\prime}, z\left(v_{k}^{\prime}\right)\right), \\
& \Psi\left(z\left(v_{i}\right), v_{k}\right)=\Psi\left(v_{i+2}^{\prime}, v_{k}\right)=-\Psi\left(v_{i+2}, v_{k}^{\prime}\right)=-\Psi\left(v_{i}, z\left(v_{k}\right)\right), \\
& \Psi\left(z\left(v_{i}\right), v_{k}^{\prime}\right)=\Psi\left(v_{i+1}, v_{k}^{\prime}\right)=-\Psi\left(v_{i}^{\prime}, v_{k+1}\right)=-\Psi\left(v_{i}, z\left(v_{k}^{\prime}\right)\right),
\end{aligned}
$$

hence the claim. Next observe that

$$
\varphi(z)^{2}=\left(\begin{array}{cc}
x & 1 \\
x^{2} & x
\end{array}\right) \cdot\left(\begin{array}{cc}
x & 1 \\
x^{2} & x
\end{array}\right)=\left(\begin{array}{cc}
2 x^{2} & 2 x \\
2 x^{3} & 2 x^{2}
\end{array}\right)=2 x \varphi(z) .
$$

Easy induction on $r$ now shows that $\varphi(z)^{r}=(2 x)^{r-1} \varphi(z)$ for all $r \in \mathbb{N}$. But then

$$
\phi(z)^{d}=(2 x)^{d-1}\left(\begin{array}{cc}
x & 1 \\
x^{2} & x
\end{array}\right)=2^{d-1}\left(\begin{array}{cc}
0 & x^{d-1} \\
0 & 0
\end{array}\right) \neq 0, \quad \varphi(z)^{d+1}=0,
$$

which implies that $z^{d} \neq 0$ and $z^{d+1}=0$. So $z$ is nilpotent and at least one part of the partition of $\operatorname{dim} M$ associated with $z$ equals $d+1$. Property 3 of our decomposition ensures that there exists $\hat{z} \in \mathfrak{g}$ with $\hat{z}_{\mid M}=z$ and $\hat{z}_{\mid V_{i}}=e_{\mid V_{i}}$ for all $i \notin\{j, j+1\}$. It is clear from the discussion above that $\hat{z} \in \mathcal{N} \cap \mathfrak{z}(e)$ and the partition of $\operatorname{dim} V$ associated with $\hat{z}$ is not dominated by that of $e$. Arguing as at the end of part 2 a of this proof we now deduce that $\hat{z} \notin \overline{G e}$, a contradiction. Thus $G$ is not of type $\mathrm{B}, \mathrm{C}$ or D. 
(3) Suppose $G$ is a group of type E. We are going to rely on some results proved in [27]. Adopt Dynkin's labelling of nilpotent orbits; see [7, Chap. 13]. Let $G_{\mathbb{C}}$ be a simple algebraic group over $\mathbb{C}$ of the same type as $G$ and let $e_{\mathbb{C}}$ be a nilpotent element in Lie $G_{\mathbb{C}}$ whose $G_{\mathbb{C}^{-}}$orbit has the same labelling as $\mathcal{O}(e) \subset \mathfrak{g}$. It follows from the main result in [33] (and also from [29, Sect. 2]) that

$$
\operatorname{dim} \mathfrak{z}(e)=\operatorname{dim}_{\mathbb{C}} \mathfrak{z}\left(e_{\mathbb{C}}\right), \quad \operatorname{dim} \mathfrak{z}(e ; 0)=\operatorname{dim}_{\mathbb{C}}\left(\mathfrak{z}\left(e_{\mathbb{C}}\right) / \operatorname{nil} \mathfrak{z}\left(e_{\mathbb{C}}\right)\right) .
$$

So we can use Elashvili's tables [7, pp. 401-407] for computing $\operatorname{dim} \mathfrak{z}(e)$ and $\operatorname{dim} \mathfrak{z}(e ; 0)$. (a) As explained in (3.2), the linear map $(\operatorname{ad} e)^{2}: \mathfrak{g}\left(-2 ; \lambda_{e}\right) \longrightarrow \mathfrak{g}\left(2 ; \lambda_{e}\right)$ is not bijective. The computations in [27] then show that $p \in\{5,7\}$ and $e$ is regular in Lie $L$ where $L$ is a Levi subgroup of $G$ with a factor of type $A_{p-1}$. There are eight such cases in all, and in each of them we have $e^{[p]}=0$.

If $G$ is of type $\mathrm{E}_{6}$ (respectively, $\mathrm{E}_{7}$ ) and $\mathcal{O}(e)$ is labelled by $\mathrm{A}_{4} \times \mathrm{A}_{1}$, then $\mathfrak{z}(e ; 0)$ is 1-dimensional (respectively, 2-dimesional); see [7, pp. 402, 404]. Combined with our earlier remarks this implies that the connected component of $Z\left(\lambda_{e}\right) \cap Z_{G}(e)$ is a torus. Hence $\mathcal{O}(e)$ is not of the above type. If $\mathcal{O}(e)$ is labelled by $\mathrm{A}_{p-1}$ and $G$ is not of type $\mathrm{E}_{7}$ when $p=7$, then $\mathfrak{z}(e)$ meets the orbit labelled by $\mathrm{A}_{p-1} \times \mathrm{A}_{1}$, call it $\mathcal{O}_{1}$. Elashvili's tables along with our earlier remarks assure that $\operatorname{dim} \mathcal{O}_{1}>\operatorname{dim} \mathcal{O}(e)$. Then $\mathcal{N} \cap \mathfrak{z}(e) \not \subset \overline{\mathcal{O}(e)}$, hence $\mathcal{O}(e)$ is not of that type.

These observations settle the case where $G$ is of type $\mathrm{E}_{6}$ and leave us with just three orbits, one in characteristic 5 and two in characteristic 7 . Unfortunately, the conclusion of Lemma 3.1 does hold for these orbits and no further reduction is readily available. So we have to work harder here, and our plan will be to exhibit, in each of the remaining cases, an element $z \in \mathcal{N} \cap \mathfrak{z}(e)$ with $z^{[p]} \neq 0$. Since $e^{[p]}=0$ and the $[p]$ th power map on $\mathfrak{g}$ is a $G$-equivariant morphism, this will imply that $\mathcal{N} \cap \mathfrak{z}(e) \not \subset \overline{\mathcal{O}(e)}$. We adopt Bourbaki's numbering of simple roots [5, Tables I-IX] and the notation of [27]. The group scheme $\left(S L_{2}\right)_{H}$ from $[27,(2.26)]$ will be denoted by $\mathcal{G}$.

(b) Suppose $p=7$ and $G$ is of type $\mathrm{E}_{8}$. Then $\mathcal{O}(e)$ is labelled by $\mathrm{A}_{6} \times \mathrm{A}_{1}$. So we may assume that $L=L_{J}$, where $J=\{1,2,4,5,6,7,8\}$, and $e=\sum_{i \in J} e_{\alpha_{i}}$ (note that $I=J$ in this case). From $[27,(2.27)]$ we know that the projective $\mathcal{G}$-module $P_{0,0}$ is a direct summand of both $\mathfrak{g}_{J}(-2)$ and $\mathfrak{g}_{J}(2)$. So $\mathfrak{z}(e ; 0) \cap \mathfrak{g}_{J}( \pm 2) \neq\{0\}$. On the other hand, $\operatorname{dim} \mathfrak{z}(e ; 0)=3$, by $[7$, p. 406]. Then $\mathfrak{z}(e ; 0)$ is a 3 -dimensional reductive Lie algebra with $\mathcal{N}(\mathfrak{z}(e ; 0)) \neq\{0\}$. Therefore, $\mathfrak{z}(e ; 0) \cong \mathfrak{s l}(2)$ as restricted Lie algebras.

Obviously, $Z\left(\lambda_{e}\right) \cap Z_{G}(e)$ contains $Z\left(L_{J}\right)$, the centre of $L_{J}$. Combined with the preceding remark this shows that there exist nonzero $e_{0} \in \mathfrak{g}_{J}(2) \cap \mathfrak{g}\left(0 ; \lambda_{e}\right), f_{0} \in$ $\mathfrak{g}_{J}(-2) \cap \mathfrak{g}\left(0 ; \lambda_{e}\right)$ and $h_{0} \in$ Lie $Z\left(L_{J}\right)$ such that $\left(e_{0}, h_{0}, f_{0}\right)$ is an $\mathfrak{s l}_{2}$-triple in $\mathfrak{z}(e ; 0)$. Since each $\mathfrak{g}_{J}(\ell)$ with $\ell \neq 0$ is an irreducible $L_{J}$-module, by $[27,(2.27)]$, the endomorphism ad $h_{0}$ acts on $\mathfrak{g}_{J}(\ell)$ as $\ell$ id.

By $[27,(2.27)]$, we have that $\mathfrak{g}_{J}( \pm 4) \cong V_{p-1,0}$ as $\mathcal{G}$-modules. In particular, the subspaces $\mathfrak{z}(e) \cap \mathfrak{g}_{J}( \pm 4)$ are 1-dimensional. Fix a nonzero $a \in \mathfrak{z}(e) \cap \mathfrak{g}_{J}(4)$, a multiple of $e_{\tilde{\alpha}}$, and put $z=f_{0}+a$. We claim that $z \in \mathcal{N}$ and $z^{[7]} \neq 0$.

For $m_{1}, \ldots, m_{s} \in \mathbb{Z}_{+}$and $n_{1}, \ldots, n_{s} \in \mathbb{N}$, set

$$
\left[a^{m_{1}} f_{0}^{n_{1}} \cdots a^{m_{s}} f_{0}^{n_{s}} a\right]:=(\operatorname{ad} a)^{m_{1}}\left(\operatorname{ad} f_{0}\right)^{n_{1}} \cdots(\operatorname{ad} a)^{m_{s}}\left(\operatorname{ad} f_{0}\right)^{n_{s}}(a) .
$$

To prove the claim we first observe that $a \in \mathfrak{g}\left(6 ; \lambda_{e}\right)$ is a primitive vector of weight 4 for $\mathfrak{z}(e ; 0)$. Hence $\left[f_{0}^{4} a\right] \neq 0$, by a standard $\mathfrak{s l}_{2}$-argument. Since $\left[f_{0}^{4} a\right]$ belongs to 
$\mathfrak{g}_{J}(-4) \cap \mathfrak{z}(e)$, a 1-dimensional subspace, it must be that

$$
\left[f_{0}^{4} a\right] \in k^{*} e_{-\delta} \quad \text { where } \delta=\underset{2}{245321} .
$$

By [15, Chap. V, Sect. 7], $z^{[7]}=a^{[7]}+f_{0}^{[7]}+\sum_{i=1}^{6} s_{i}\left(a, f_{0}\right)$ where

$$
\operatorname{ad}\left(t a+f_{0}\right)^{6}(a)=\sum_{i=1}^{6} i s_{i}\left(a, f_{0}\right) t^{i-1} \quad(\forall t \in k) .
$$

Since $\mathfrak{g}_{J}(6)$ is zero, we have $\left[a f_{0} a\right]=\left[a^{2} f_{0}^{3} a\right]=0$. Then $\left[a f_{0}^{2} a\right]=0$, by the Jacobi identity. It follows that

$$
\operatorname{ad}\left(t a+f_{0}\right)^{6}(a)=\operatorname{ad}\left(t a+f_{0}\right)^{3}\left(\left[f_{0}^{3} a\right]\right)=\operatorname{ad}\left(t a+f_{0}\right)^{2}\left(t\left[a f_{0}^{3} a\right]+\left[f_{0}^{4} a\right]\right) .
$$

As $\mathfrak{g}_{J}(-6)$ is zero, $\left[f_{0}^{5} a\right]=0$ necessarily holds. Since both $\left[a^{2} f_{0}^{4} a\right]$ and $\left[a f_{0} a f_{0}^{3} a\right]$ belong to $\mathfrak{g}_{J}(4) \cap \mathfrak{g}\left(18 ; \lambda_{e}\right)$, we also have $\left[a^{2} f_{0}^{4} a\right]=\left[a f_{0} a f_{0}^{3} a\right]=0$. So (12) yields

$$
\operatorname{ad}\left(t a+f_{0}\right)^{6}(a)=t\left[t a+f_{0},\left[a f_{0}^{4} a\right]+\left[f_{0} a f_{0}^{3} a\right]\right]=t\left(\left[f_{0} a f_{0}^{4} a\right]+\left[f_{0}^{2} a f_{0}^{3} a\right]\right) .
$$

Now let $F=\operatorname{ad} f_{0}$ and $A=\operatorname{ad} a$. Since $\left[f_{0}^{5} a\right]=0$ we have $(\operatorname{ad} F)^{5}(A)$ forcing

$$
\left(F^{5} A-5 F^{4} A F+10 F^{3} A F^{2}-10 F^{2} A F^{3}+5 F A F^{4}-A F^{5}\right)(a)=0 .
$$

Together with our observations above this gives $\left[f_{0} a f_{0}^{4} a\right]=2\left[f_{0}^{2} a f_{0}^{3} a\right]$. By (13),

$$
\operatorname{ad}\left(t a+f_{0}\right)^{6}(a)==3 t\left[f_{0}^{2} a f_{0}^{3} a\right]=-4 t\left[f_{0}^{2} a f_{0}^{3} a\right]=2 s_{2}\left(a, f_{0}\right) t .
$$

So $s_{i}\left(a, f_{0}\right)=0$ for $i \neq 2$. Also, $f_{0}^{[7]}=a^{[7]}=0$, because $\mathfrak{z}(e ; 0) \cong \mathfrak{s l}(2)$ as restricted Lie algebras. It follows that

$$
z^{[7]}=s_{2}\left(a, f_{0}\right)=-2\left[f_{0}^{2} a f_{0}^{3} a\right] \in \mathfrak{g}_{J}(-2) \subset \mathcal{N} .
$$

According to $\left[5\right.$, Table VIII], $\tilde{\alpha}-\delta$ is a root. So (11) yields $\left[a f_{0}^{4} a\right] \neq 0$. This, in turn, implies that $\left[a f_{0}^{3} a\right] \neq 0$. Indeed, otherwise

$$
\left[a f_{0}^{4} a\right]=\left[\left[a f_{0}\right],\left[f_{0}^{3} a\right]\right]=\left[f_{0},\left[\left[a, f_{0}\right],\left[f_{0}^{2} a\right]\right]\right]=\left[f_{0},\left[a f_{0}^{3} a\right]\right]=0,
$$

a contradiction (here we used the Jacobi identity and the fact that $\left[a f_{0}^{2} a\right]=0$ ). Since $\left[e_{0},\left[a f_{0}^{3} a\right]\right] \in k\left[a f_{0}^{2} a\right]$, we obtain that $\left[a f_{0}^{3} a\right]$ is a primitive vector of weight 2 for $\mathfrak{z}(e ; 0)$. But then $\left[f_{0}^{2} a f_{0}^{3} a\right] \neq 0$, by a standard $\mathfrak{s l}_{2}$-argument. Now(14) shows that $z \in \mathcal{N}$ and $z^{[7]} \neq 0$, as claimed. This settles the present case.

(c) Retain the assumptions of part 3b. Since $G$ is of type $\mathrm{E}_{8}$, it is both adjoint and simply connected. In particular, there is a unique involution $\tau \in T \subset G$ with the property that that $(\operatorname{Ad} \tau) x=(-1)^{\ell} x$ for all $x \in \mathfrak{g}_{J}(\ell)$ and all $\ell \in \mathbb{Z}$. Let $G^{\tau}=Z_{G}(\tau)$ and let $\mathfrak{g}^{\tau}$ be the fixed point algebra of $\operatorname{Ad} \tau$. By a result of Steinberg, $G^{\tau}$ is a connected reductive group; see [35, Chap. II, § 4]. By [4, Chap. 3, Sect. 9], we have $\mathfrak{g}^{\tau}=$ Lie $G^{\tau}$.

Clearly, $T \subset G^{\tau}$. Straightforward calculation shows that $\operatorname{dim} \mathfrak{g}^{\tau}=136$. Since all maximal root subsystems in the root system of type $\mathrm{E}_{8}$ are well-known (see [5, Chap. VI, $\S 4$, Exercise 4] for example), one finds out without difficulty that $G^{\tau}$ is a semisimple group of type $\mathrm{E}_{7} \times \mathrm{A}_{1}$. Let $G_{1}$ and $G_{2}$ be the simple components of $G^{\tau}$ of type $\mathrm{E}_{7}$ and $\mathrm{A}_{1}$, respectively, and $\mathfrak{g}_{i}=$ Lie $G_{i}$. The Lie algebras $\mathfrak{g}_{1}$ and $\mathfrak{g}_{2}$ are simple, hence $\mathfrak{g}^{\tau}=\mathfrak{g}_{1} \oplus \mathfrak{g}_{2}$, a direct sum of restricted Lie algebras.

Given $x \in \mathfrak{g}^{\tau}$ we let $x_{i}$ be the component of $x$ in $\mathfrak{g}_{i}$. Then $x^{[7]}=x_{1}^{[7]}+x_{2}^{[7]}$. Since $\mathfrak{g}_{2} \cong \mathfrak{s l}(2)$, we also have that $x_{2}^{[7]}=0$ for any $x \in \mathfrak{g}^{\tau} \cap \mathcal{N}$. Note that $e, z \in \mathfrak{g}^{\tau}$. The preceding remark shows that $e_{1}^{[7]}=0$ and $z_{1}^{[7]} \neq 0$. Obviously, $\left[e_{1}, z_{1}\right]=0$. 
Next observe that $P_{J} \cap G^{\tau}$ is a parabolic subgroup of $G^{\tau}$ and $L_{J}$ is a Levi subgroup in it. From this it follows that $L_{J} \cap G_{1}$ is a Levi subgroup of type $A_{6}$ in $G_{1}$. Since $e$ is regular in $\mathfrak{l}_{J}$, its component $e_{1}$ must be regular in $\mathfrak{l}_{J} \cap \mathfrak{g}_{1}=\operatorname{Lie}\left(L_{J} \cap G_{1}\right)$. By the discussion above, $z_{1} \notin \overline{\left(\operatorname{Ad} G_{1}\right) e_{1}}$. This settles our second remaining case in characteristic 7 .

(d) Now suppose $p=5$. Then $G$ is of type $\mathrm{E}_{7}$ and $\mathcal{O}(e)$ is labelled by $\mathrm{A}_{4} \times \mathrm{A}_{2}$; see part 3a. So we may assume that $L=L_{J}$, where $J=\{1,2,3,4,6,7\}$, and $e=\sum_{i \in J} e_{\alpha_{i}}$. Since $\tilde{\alpha}=\begin{gathered}234321 \\ 2\end{gathered}$, we have $M_{J,+}=\mathfrak{g}_{J}(1) \oplus \mathfrak{g}_{J}(2) \oplus \mathfrak{g}_{J}(3)$. Using [5, Table VI] we observe that $\mathfrak{g}_{J}(1), \mathfrak{g}_{J}(2)$ and $\mathfrak{g}_{J}(3)$ are irreducible modules over $\left(L_{J}, L_{J}\right) \cong \mathrm{SL}_{5}(k) \times$ $\mathrm{SL}_{3}(k)$ with highest weights $\omega_{3}^{J}+\omega_{7}^{J}, \omega_{2}^{J}+\omega_{6}^{J}$ and $\omega_{1}^{J}$, respectively. As a consequence,

$$
\mathfrak{g}_{J}(1) \cong\left(\stackrel{2}{\wedge} N_{5}\right) \otimes N_{3}, \quad \mathfrak{g}_{J}(2) \cong N_{5}^{*} \otimes N_{3}, \quad \mathfrak{g}_{J}(3) \cong N_{5}
$$

As in $[27,(2.27)]$ this yields that each $\mathfrak{g}_{J}(\ell)$ with $\ell \neq 0$ is a projective $\mathcal{G}$-module. Direct calculation based on [5, Table VI] and [27, Lemma 2.9] gives $m_{1}(e)=8$, $m_{2}(e)=6, m_{3}(e)=4$ showing that $2 p-2$ is the largest $\lambda_{e}$-weight of $M_{J,+}$. Then $[27,(2.26)]$ says that all indecomposable summands of the $\mathcal{G}$-modules $M_{J, \pm}$ are of the form $V_{p-1,0}$ or $P_{m, 0}$ for $m \leq p-2$. Moreover, $P_{0,0}$ occurs in $\mathfrak{g}_{J}( \pm 1)$.

On the other hand, $\operatorname{dim} \mathfrak{z}(e ; 0)=3$, by [7, p. 406]. Arguing as in part $3 \mathrm{~b}$ we now obtain that there are nonzero $e_{0} \in \mathfrak{g}_{J}(1) \cap \mathfrak{g}\left(0 ; \lambda_{e}\right), f_{0} \in \mathfrak{g}_{J}(-1) \cap \mathfrak{g}\left(0 ; \lambda_{e}\right)$ and $h_{0} \in$ Lie $Z\left(L_{J}\right)$ such that $\left(e_{0}, h_{0}, f_{0}\right)$ is an $\mathfrak{s l}_{2}$-triple in $\mathfrak{z}(e ; 0)$. Since each $\mathfrak{g}_{J}(\ell)$ with $\ell \neq 0$ is $L_{J}$-irreducible, ad $h_{0}$ acts on $\mathfrak{g}_{J}(\ell)$ as $2 \ell \mathrm{Id}$ (because $e_{0} \in \mathfrak{g}_{J}(1)$ and $\left.\left[h_{0}, e_{0}\right]=2 e_{0}\right)$. As in part $3 \mathrm{~b}, \mathfrak{z}(e ; 0) \cong \mathfrak{s l}(2)$ as restricted Lie algebras.

Since $\mathfrak{g}_{J}(-3)^{*} \cong \mathfrak{g}_{J}(3) \cong N_{5}$ as $\left(L_{J}, L_{J}\right)$-modules and since $e$ is regular nilpotent in $\mathfrak{l}_{J}$, it must be that $\mathfrak{g}_{J}( \pm 3) \cong V_{p-1,0}$ as $\mathcal{G}$-modules. In particular, the subspaces $\mathfrak{z}(e) \cap \mathfrak{g}_{J}( \pm 3)$ are 1-dimensional. Fix a nonzero $a \in \mathfrak{z}(e) \cap \mathfrak{g}_{J}(3)$, an element in $\mathfrak{g}\left(4 ; \lambda_{e}\right)$, and put $z=f_{0}+a$. We claim that $z \in \mathcal{N}$ and $z^{[5]} \neq 0$.

By [15, Chap. V, Sect. 7], $z^{[5]}=a^{[5]}+f_{0}^{[5]}+\sum_{i=1}^{4} s_{i}\left(a, f_{0}\right)$ where

$$
\operatorname{ad}\left(t a+f_{0}\right)^{4}(a)=\sum_{i=1}^{4} i s_{i}\left(a, f_{0}\right) t^{i-1} \quad(\forall t \in k) .
$$

Since $\mathfrak{g}_{J}(5)$ is zero, we have $\left[a f_{0} a\right]=0$. Then $\left[a f_{0}^{2} a\right]=0$, by the Jacobi identity. Furthermore, $\left[a f_{0}^{3} a\right]=0$ as $\mathfrak{g}_{J}(3) \cap \mathfrak{g}\left(8 ; \lambda_{e}\right)$ is zero. Hence

$$
\operatorname{ad}\left(t a+f_{0}\right)^{4}(a)=\operatorname{ad}\left(t a+f_{0}\right)\left(\left[f_{0}^{3} a\right]\right)=\left[f_{0}^{4} a\right]=s_{1}\left(a, f_{0}\right) .
$$

This means that $s_{i}\left(a, f_{0}\right)=0$ for $i>1$. Since $a^{[5]}=f_{0}^{[5]}=0$, we now get

$$
z^{[5]}=\left[f_{0}^{4} a\right] \in \mathfrak{g}_{J}(-1) \subset \mathcal{N} .
$$

Since $a$ is a primitive vector of weight 1 for $\mathfrak{z}(e ; 0)$, the element $\left(\operatorname{ad} e_{0}\right)^{2}\left(\left[f_{0}^{4} a\right]\right)$ is a nonzero multiple of $\left[f_{0}^{2} a\right]$. Due to $(15)$ we are thus reduced to show that $\left[f_{0}^{2} a\right] \neq 0$.

Since $\beta_{J}^{5}={ }_{1}^{122111}$ and $b_{J}^{5}=10$, it follows from [27, Lemma 2.9] and [5, Table VI] that the subspace $\mathfrak{g}_{J}(-1) \cap \mathfrak{g}\left(0 ; \lambda_{e}\right)$ is spanned by $e_{-\gamma_{1}}, \ldots, e_{-\gamma_{6}}$ where

$$
\begin{aligned}
& \gamma_{1}=\begin{array}{c}
001111 \\
1
\end{array}, \quad \gamma_{2}=\begin{array}{c}
01111 \\
0
\end{array}, \quad \gamma_{3}=\begin{array}{c}
11110 \\
0
\end{array}, \\
& \gamma_{4}=\begin{array}{c}
01110 \\
1
\end{array}, \quad \gamma_{5}={ }_{23}^{111100}, \quad \gamma_{6}=\begin{array}{c}
012100 \\
1
\end{array}
\end{aligned}
$$


Let $\beta=\sum_{i=1}^{7} \alpha_{i}$, a positive root. We may assume without loss of generality that

$$
\begin{aligned}
& e_{-\gamma_{1}}=\left[e_{\alpha_{3}},\left[e_{\alpha_{1}}, e_{-\beta}\right]\right], \quad e_{-\gamma_{2}}=\left[e_{\alpha_{2}},\left[e_{\alpha_{1}}, e_{-\beta}\right]\right], \quad e_{-\gamma_{3}}=\left[e_{\alpha_{2}},\left[e_{\alpha_{7}}, e_{-\beta}\right]\right] \\
& e_{-\gamma_{4}}=\left[e_{\alpha_{7}},\left[e_{\alpha_{1}}, e_{-\beta}\right]\right], \quad e_{-\gamma_{5}}=\left[e_{\alpha_{6}},\left[e_{\alpha_{7}}, e_{-\beta}\right]\right], \quad\left[e_{\alpha_{4}}, e_{-\gamma_{6}}\right]=\left[e_{\alpha_{1}}, e_{-\gamma_{5}}\right] .
\end{aligned}
$$

Let $f_{0}=\sum_{i=1}^{6} s_{i} e_{-\gamma_{i}}$ where $s_{i} \in k$. Recall that $\left[e, f_{0}\right]=0$ and $e=\sum_{i \neq 5} e_{\alpha_{i}}$. Since $\left[e_{\alpha_{2}}, e_{\alpha_{3}}\right]=\left[e_{\alpha_{3}}, e_{\alpha_{7}}\right]=\left[e_{\alpha_{2}}, e_{\alpha_{6}}\right]=0$, this yields $s_{1}+s_{2}=s_{1}+s_{4}=s_{3}+s_{5}=0$. Since $e_{\alpha_{1}}, e_{\alpha_{2}}, e_{\alpha_{7}}$ pairwise commute we also have $s_{2}+s_{3}+s_{4}=0$. Since $\left[e_{\alpha_{1}}, e_{\alpha_{6}}\right]=0$, our choice of $e_{-\gamma_{6}}$ yields $s_{4}+s_{5}+s_{6}=0$.

Together these relations show that the $s_{i}$ 's are all nonzero. Since $\mathfrak{g}_{J}(3) \cong V_{p-1,0}$ as $\mathcal{G}$-modules, we have $a \in k^{*} e_{\tilde{\alpha}}$. In view of [5, Table VI] we also have

$$
\left[f_{0}, e_{\tilde{\alpha}}\right] \in\left\{ \pm s_{3} e_{1232}^{2} 2_{2} 11 \pm s_{5} e_{1232}{ }_{1} 21\right\} \text {. }
$$

Using [5, Table VI] one now observes without difficulty that $\left[f_{0}^{2} a\right]$ is a nonzero linear combination of $e_{0}{ }_{1}^{2111}, e_{1} 1111, e_{1}{ }_{1} 110$. Thus $G$ is not of type E.

(4) Next suppose $G$ is of type $\mathrm{F}_{4}$. Then $G=\operatorname{Aut}(\mathfrak{g})$. Let $\tilde{G}$ be a simply connected group of type $\mathrm{E}_{6}$ and $\tilde{\mathfrak{g}}=$ Lie $\tilde{G}$. As in $[27,(2.28)]$, we regard $\mathfrak{g}$ as a subalgebra of $\tilde{\mathfrak{g}}$. More precisely, we assume that $\mathfrak{g}=\tilde{\mathfrak{g}}^{\sigma}$ where $\sigma$ is the involution in $\operatorname{Aut}(\tilde{\mathfrak{g}})$ swapping $e_{ \pm \alpha_{i}}$ and $e_{ \pm \alpha_{-i+7}}$ for $i=1,2$ and fixing $e_{ \pm \alpha_{i}}$ for $i=2,4$. Note that $\sigma$ permutes the set of indices $\{1,2, \ldots, 6\}$.

Suppose $e$ is conjugate to a regular nilpotent element in a standard Levi subalgebra of $\mathfrak{g}$. Then a $G$-conjugate of $e$ is regular in a standard Levi subalgebra $\mathfrak{l}_{\tilde{J}}$ of $\tilde{\mathfrak{g}}$ such that $\tilde{J}^{\sigma}=\tilde{J}$; see $[27,(2.28)]$. Hence it can be assumed in the present case that $e=\sum_{i \in \tilde{J}} e_{\alpha_{i}}$. Let $\tilde{\lambda}$ denote the image of $\lambda_{\tilde{J}, \tilde{J}} \in X_{*}(\tilde{G})$ under the natural embedding $X_{*}(\tilde{G}) \hookrightarrow X_{*}(\operatorname{Aut}(\tilde{\mathfrak{g}}))$. It is easy to see that $\sigma$ fixes $\tilde{\lambda}$.

The construction of optimal 1-parameter subgroups in [29, Sect. 2] shows that we can take for $\lambda_{e}$ the image of $\tilde{\lambda}$ in $X_{*}(G)=X_{*}\left(\operatorname{Aut}\left(\tilde{\mathfrak{g}}^{\sigma}\right)\right)$. Since $\mathfrak{l}_{\tilde{J}}$ is $\sigma$-stable and $p>3$, the root system of $\mathfrak{l}_{\tilde{J}}$ has no components of type $\mathrm{A}_{p-1}$. Calculations in [27] then yield that the map $(\operatorname{ad} e)^{2}: \tilde{\mathfrak{g}}(-2 ; \tilde{\lambda}) \longrightarrow \tilde{\mathfrak{g}}(2 ; \tilde{\lambda})$ is bijective. It follows that so is the map $(\operatorname{ad} e)^{2}: \mathfrak{g}\left(-2 ; \lambda_{e}\right) \longrightarrow \mathfrak{g}\left(2 ; \lambda_{e}\right)$.

If $e$ is not of the above type, then it follows from $[27,(2.28)]$ and the proof of $[27$, Lemma 2.7] that the map $(\operatorname{ad} e)^{2}: \mathfrak{g}\left(-2 ; \lambda_{e}\right) \longrightarrow \mathfrak{g}\left(2 ; \lambda_{e}\right)$ is bijective. This shows that $G$ is not of type $\mathrm{F}_{4}$.

(5) Finally, suppose $G$ is of type $\mathrm{G}_{2}$. Note that $e \neq 0$ and $e$ is not conjugate to a long root vector in $\mathfrak{g}$ (otherwise $\mathfrak{z}(e)$ would contain a regular nilpotent element in $\mathfrak{g}$ ). By [7, p. 401], we are now left with the orbit labelled by $\tilde{\mathrm{A}}_{1}$. So suppose $e=e_{\alpha_{2}}$. Then $I=J=\{2\}$ and we may assume further that $\lambda_{e}=\alpha_{2}^{\vee}$. It is easily seen that in this case $\mathfrak{z}(e ; 0) \cong \mathfrak{s l}(2)$ and $\operatorname{dim} \mathfrak{g}\left( \pm 2 ; \lambda_{e}\right)=1$. But then Lemma 3.1 shows that this case is impossible, completing the proof of Proposition 3.2.

\section{REFERENCES}

[1] R. Basili, On the irreducibility of commuting varieties of nilpotent matrices, J. Algebra, to appear. arXive:math.AG/0301215v1.

[2] V. Baranovsky, The variety of pairs of commuting nilpotent matrices is irreducible, Transform. Groups 6 (2001) 3-8. 
[3] P. Bardsley and R.W. Richarson, Étale slices for algebraic transformation groups in characteristic p, Proc. London Math. Soc. 51 (1985) 295-317.

[4] A. Borel, Linear algebraic groups, Benjamin, New York, 1969.

[5] N. Bourbaki, Groupes et algèbres de Lie, Chapitres IV, V, VI, Hermann, Paris, 1968.

[6] J. Briançon, Description de Hilb ${ }^{n} \mathbb{C}\{x, y\}$, Invent. Math. 41 (1977) 45-89.

[7] R.W. Carter, Finite groups of Lie type, conjugacy classes and complex characters, Wiley, Chichester and New York, 1985.

[8] J. Fogarty, Algebraic families on an algebraic surface, Amer. J. Math. 10 (1968) 511-521.

[9] J. Fogarty, Algebraic families on an algebraic surface II: Picard scheme of the punctual Hilbert scheme, Amer. J. Math. 96 (1974) 660-687.

[10] M. Gerstenhaber, Dominance over the classical groups, Ann. of Math. 74 (1961) 532-569.

[11] M. Granger, Géométrie des schémas de Hilbert ponctuels, Mém. Soc. Math. France, n. 8, 1983.

[12] D. Gross, Higher nullcones and commuting varieties, Comm. Algebra 21 (1993) 1427-1455.

[13] D.F. Holt and N. Spaltenstein, Nilpotent orbits of exceptional Lie algebras over algebraically closed fields of bad characteristic, J. Austral. Math. Soc. 38 (1985) 330-350.

[14] A. Iarrobino, Punctual Hilbert schemes, Mem. Amer. Math. Soc., Vol. 188, 1977.

[15] N. Jacobson, Lie algebras, Interscience, New York, 1962.

[16] J.C. Jantzen, Nilpotent orbits in representation theory, 2001, Notes from Odense summer school, August 2000.

[17] A. Joseph, On a Harish-Chandra homomorphism, C.R. Acad. Sci. Paris Sér. I Math. 324 (1997) $759-764$.

[18] H. Kraft, Geometrische Methoden in der Invariantentheorie, Aspekte Math. D1, Vieweg, Braunschweig-Wiesbaden, 1984.

[19] P. Levy, Commuting varieties of Lie algebras over fields of prime characteristic, J. Algebra 250 (2002) 473-484.

[20] G. Lusztig and N. Spaltenstein, Induced unipotent classes, J. London Math. Soc. (2) 19 (1979) $41-52$.

[21] G. McNinch, Sub-principal homomorphisms in positive characteristic, Math. Zeitschrift, to appear. arXive:math.RT/0108140.

[22] H. Nakajima, Lectures on Hilbert schemes of points on surfaces, University Lecture Series, Vol. 18, Amer. Math. Soc., Providence, 1999.

[23] K. Pommerening, Über die unipotenten Klassen reduktiver Gruppen, J. Algebra 49 (1977) 525536.

[24] K. Pommerening, Über die unipotenten Klassen reduktiver Gruppen, II, J. Algebra 65 (1980) 373-398.

[25] A. Premet, On Cartan subalgebras of Lie p-algebras, Izv. Acad. Nauk SSSR Ser. Mat. 50 (1986) 788-800 (Russian). Math. USSR-Izv. 29 (1987) 135-159 (English transl.)

[26] A. Premet, Regular Cartan subalgebras and nilpotent elements in restricted Lie algebras, Mat. Sb. 180 (1989) 542-557 (Russian). Math. USSR-Sb. 66 (1990) 555-570 (English transl.)

[27] A. Premet, An analogue of the Jacobson-Morozov theorem for Lie algebras of reductive groups of good characteristics, Trans. Amer. Math. Soc. 347 (1995) 2961-2988.

[28] A. Premet, Complexity of Lie algebra representations and nilpotent elements of the stabilizers of linear forms, Math. Z. 228 (1998) 255-282.

[29] A. Premet, Nilpotent orbits in good characteristic and the Kempf-Rousseau theory, J. Algebra 260 (2003) 338-366.

[30] R.W. Richardson, Conjugacy classes in Lie algebras and algebraic groups, Ann. of Math. 86 (1967) $1-15$.

[31] R.W. Richardson, Commuting varieties of semisimple Lie algebras and algebraic groups, Compositio Math. 38 (1979) 311-327.

[32] I.R. Shafarevich, Basic algebraic geometry, 2nd ed. Vol. 1, Springer-Verlag, Berlin-HeidelbergNew York, 1994.

[33] N. Spaltenstein, Existence of good transverse slices to nilpotent orbits in good characteristic, J. Fac. Sci. Univ. Tokyo (IA Math) 30 (1984) 283-286. 
[34] T.A. Springer, Some arithmetical results on semi-simple Lie algebras, Publ. Math. IHES 30 (1966) 115-141.

[35] T.A. Springer and R. Steinberg, Conjugacy classes, In Seminar on algebraic groups and related topics, Lecture Notes in Math.,Vol. 131, Springer-Verlag, Berlin-Heidelberg-New York, 1970, pp. $167-266$.

[36] R. Steinberg, Conjugacy classes in algebraic groups, Lecture Notes in Math., 366, SpringerVerlag, Berlin-Heidelberg-New York, 1974.

[37] A. Suslin, E. Friedlander and C. Bendel, Infinitesimal 1-parameter subgroups and cohomology, J. Amer. Math. Soc. 10 (1997) 693-728.

Department of Mathematics, University of Manchester, Oxford Road, M13 9PL, $\mathrm{UK}$

E-mail address: sashap@ma.man.ac.uk 